\title{
Ancient Stardust in Fine-grained Chondrule Dust Rims from Carbonaceous Chondrites
}

Authors: Jan Leitner ${ }^{1}$, Christian Vollmer ${ }^{2}$, Christine Floss $^{3}$, Jutta Zipfel ${ }^{4}$, Peter Hoppe ${ }^{1}$

${ }^{1}$ Max Planck Institute for Chemistry, Particle Chemistry Department, Hahn-Meitner-Weg 1, 55128 Mainz, Germany.

${ }^{2}$ Institute for Mineralogy, Westfälische Wilhelms-Universität, Correnstrasse 24, 48149 Münster, Germany.

${ }^{3}$ Laboratory for Space Sciences and Physics Department, Washington University, One Brookings Drive, St. Louis, MO 63130, USA.

${ }^{4}$ Forschungsinstitut und Naturmuseum Senckenberg, Sektion Meteoritenforschung, Senckenberganlage 25, 60325 Frankfurt, Germany.

*Correspondence to: jan.leitner@mpic.de

\author{
Submitted to \\ Earth and Planetary Science Letters
}

March 26, 2015

Redrafted version submitted

May 5, 2015

Revised version submitted

September 06, 2015

Second revised version submitted

November 09, 2015 


\section{ABSTRACT}

2 Carbonaceous chondrites are fragments from primitive parent asteroids, which represent some

3 of the most primitive meteorites accessible for laboratory analysis and offer therefore the best 4 opportunity to explore the chemical and physical conditions in the early Solar System. Here, 5 we report the identification of presolar grains, which are circumstellar condensates that date 6 back from before the formation of our Solar System, in fine-grained dust rims around 7 chondrules in carbonaceous chondrites. Average presolar grain abundances in the rims of 8 aqueously altered chondrites (petrologic type 2) are three times higher than in the respective 9 interchondrule matrices, while for the most pristine specimens (petrologic type 3), the

Keywords: carbonaceous chondrites; chondrule rims; Solar Nebula; NanoSIMS; circumstellar matter 


\section{INTRODUCTION}

Primitive meteorites, interplanetary dust particles (IDPs), and cometary matter contain small but significant amounts of refractory dust grains with highly anomalous isotopic compositions. The observed isotopic anomalies cannot be explained by chemical or physical processes occurring in the solar system, but are the result of nucleosynthetic reactions in stellar environments. These grains formed in the winds of evolved stars and in the ejecta of stellar explosions, were incorporated in the protosolar nebula and survived alteration and homogenization processes during the formation of the solar system. They represent samples of ancient stardust available for laboratory analysis (e.g., ZINNER 2014). Investigation of the isotopic compositions and mineralogies of these grains provides valuable information on stellar nucleosynthesis and evolution, grain growth in circumstellar environments, the types of stars that contributed material to our planetary system, and chemical and physical processes in the interstellar medium and in the early Solar System. Moreover, parent body (i.e., asteroidal) alteration processes can be studied by using presolar grain abundances as tracers, as is the case for the present study.

Refractory silicates are the most abundant presolar grain type, if nanodiamonds, whose abundances and origins are a matter of ongoing discussion, are neglected (ZINNER 2014)..

Matrix-normalized abundances depend on the degree of secondary alteration experienced by the host material, but can exceed $200 \mathrm{ppm}$ in the most primitive meteorites (e.g., NGUYEN et al. 2007; Floss \& StADERMANN 2009; VOLLMER et al. 2009a; NitTLER et al. 2013). Presolar oxides are somewhat less abundant, with abundances of up to tens of ppm in meteorites (e.g., VOLLMER et al. 2009a; LEITNER et al. 2012). The majority of presolar O-rich grains are divided into four distinct groups, according to their oxygen isotopic compositions (NITTLER et al. 1997, 2008). These groups reflect different stellar origins, with major contributions from low-mass red giant stars and smaller but still significant contributions from supernovae. 
In past investigations, the fine-grained matrix material hosting the larger meteorite components (ICM - interchondrule matrix - hereafter) has been targeted for presolar grain searches. However, further fine-grained components are also present in primitive meteorites, such as fine-grained rims (FGR) around chondrules and fine-grained lithic clasts with distinct boundaries embedded in surrounding material. The origin and nature of fine-grained rims around chondrules has been and is still discussed controversially. Both nebular (e.g., ALLEN et al. 1980; MeTZler et al. 1992; Ciesla et al. 2003; ChIZMADiA \& BREARLEy 2008; Bland et al. 2011) and parent body (e.g., SeARS et al. 1993; TOMEOKA \& TANIMURA 2000; TRIGORODRIGUEZ et al. 2006) formation processes have been suggested, based on a wide variety of observations. If the FGRs accreted in a nebular setting, relatively pristine material like stardust grains or isotopically anomalous organic material could have been preserved there, possibly modified by parent body alteration. Parent body scenarios include chondrule erosion or accretion in the regolith. However, not all structures described as "fine-grained rims" are alike. The FGRs studied in this paper (Figure 1, Table S1), as well as those investigated in CO (HAENECOUR \& Floss 2012; DAVIDSON et al. 2012; HAENECOUR et al. 2014) and CM chondrites (e.g., METZLER et al. 1992; ChIZMADIA \& BREARLEY 2008; LEITNER et al. 2013) share certain properties like the co-existence of primitive, nearly unaltered components with constituents that have undergone aqueous alteration, on small scales (micrometer to submicrometer range). In contrast, the rims described by TOMEOKA \& TANIMURA (2000) and TAKAYAMA \& TOMEOKA (2012) in several CV chondrites and the Tagish Lake meteorite, have been affected heavily by aqueous alteration and may well have a different origin. The CR (Renazzo-type) group of carbonaceous chondrites is considered to be among the most primitive meteorites (KROT et al. 2002). They exhibit bulk ${ }^{15} \mathrm{~N}$ enrichments with $\delta^{15} \mathrm{~N}$ between $+20 \%$ and $+230 \%$ (depending on weathering grade and state of alteration), hosted in isotopically anomalous organic matter found in these meteorites. Individual isotopic 
hotspots display ${ }^{15} \mathrm{~N}$ enrichments of up to a factor of three (BUSEMANN et al. 2006; ALEXANDER et al. 2007). The degree of aqueous alteration observed within the CR group varies from heavily altered to very little alteration. There is an ongoing debate whether the least altered CR chondrites (MET 00426, QUE 99177) are of petrologic type 2 or 3; however, recently found evidence for aqueous alteration (LE GUILLOU \& BREARLEY 2014) would speak against a classification as type 3 . A more sensitive scale for the degree of aqueous alteration experienced by $\mathrm{CR}$ chondrites is desirable, since such information would ultimately allow differences in the presolar grain abundances due to differing degrees of alteration to be disentangled from inherent heterogeneities. Several approaches have been undertaken to establish more refined classification schemes (ALEXANDER et al. 2013; HARJU et al. 2014; HowARD et al. 2015). However, these approaches have not yet provided a petrological classification that reflects the vast differences in presolar grain abundances and distributions observed for the CR chondrites (Figure S2). For more extensively altered meteorites, the scale devised by HARJU et al. (2014) is consistent with the significantly lower presolar silicate and oxide abundances observed for Renazzo. However, ALEXANDER et al. (2013) assigned a subtype to Renazzo which puts it in line with the most pristine CR chondrites, indicating less aqueous metamorphism than in QUE 99177. Similar problems are encountered with the scheme by Howard et al. (2015). Here, MET 00426 is assigned 2.6, while QUE 99177, GRA 95229 and even GRA 06100 are classified as 2.8. There are several possible explanations for this apparent discrepancy. First of all, heterogeneous alteration within the CRs might be an important issue. Second, variations of the alteration temperatures could have affected the fraction of surviving presolar silicates and oxides (ALEXANDER et al. 2013). Finally, TEM studies have found that the amorphous silicates in the minimally altered CR chondrite MET 00426 contain water (LE GUILLOU \& BREARLEY 2014). These authors ascribe their findings to the simultaneous accretion of dust and ice. The latter produced localized centers of aqueous alteration on sublimation, offering an explanation for the survival of larger amounts of 
presolar grains in MET 00426 than in the CR2 chondrites (which therefore might have experienced alteration at higher temperatures).

Bulk O-isotopic data for the fine-grained matrix of a set of CR chondrites (SCHRADER et al. 2014) seem to correlate quite well with both the degree of alteration of the respective meteorite and the abundances of presolar O-anomalous grains from the current study (Figure S3). However, there is (yet) only small overlap between the sample sets, and heterogeneous alteration also has to be taken into account, as can be seen from differing results for individual samples from the same specimen. Therefore, we refrained from adopting any of these classification schemes for the meteorites studied here.

Early studies of CR chondrites indicated only low presolar O-anomalous dust abundances (NAGASHIMA et al. 2004; FlOSS \& STADERMANN 2005). However, more recent investigations (Floss \& StADERMANN 2009; LEITNER et al. 2012; NGUYEN et al. 2010; ZHAO et al. 2013) have revealed much higher oxygen-rich presolar dust concentrations in several of these meteorites. CR chondrites offer the opportunity to study the effects of alteration on primitive meteoritic material from a common reservoir in the early solar nebula. It has been suggested that the range of presolar silicate concentrations among different meteorites is due to secondary alteration processes (e.g., FLOSS \& STADERMANN 2009), which destroy presolar silicate grains preferentially over more refractory presolar phases such as oxides or carbonaceous grains, as has been earlier discussed by Huss et al. (2003).

Here, we report the identification of oxygen-rich presolar grains in fine-grained chondrule rims of several CR chondrites, as well as the ungrouped carbonaceous chondrite Acfer 094. The presence of stardust, together with other primitive materials, indicates a nebular origin of the fine-grained material of which the chondrule rims are comprised. Moreover, formation of these rims must have occurred prior to the formation of the respective meteorite parent bodies. Thus, our findings give strong support to nebular formation scenarios. 


\section{MATERIALS AND METHODS/EXPERIMENTAL}

\subsection{Sample characterization}

124

125

126

All investigated samples (Table1) were characterized by backscatter electron (BSE) imaging and X-ray elemental mapping with a LEO 1530 Field Emission Scanning Electron Microscope (FE-SEM) equipped with an Oxford INCA/Aztec EDS system at the Max Planck Institute for Chemistry (MPIC). Additional element distribution maps were acquired with a JEOL Superprobe 8200 Electron Microprobe at the MPI for Chemistry. Rim structures were identified either from BSE images or X-ray element maps (see Figure 1 for an example).

\subsection{Isotopic Measurements by NanoSIMS}

Suitable regions of these fine-grained materials were selected for analysis of O-isotopic compositions with the NanoSIMS 50 ion probe at the MPIC in Mainz. In addition, $\mathrm{C}$ and $\mathrm{N}$ isotopes were measured on a subset of fine-grained material (one FGR in GRA 95229, one FGR in EET 92161, and interchondrule matrix areas in GRA 95229). All isotope measurements were performed by ion imaging with a primary $\mathrm{Cs}^{+}$beam $(100 \mathrm{~nm}$ diameter, $\sim 1$ pA) which was rastered over $10 \times 10 \mu \mathrm{m}^{2}$-sized sample areas. For a more detailed description of applied data reduction and correction procedures see LEITNER et al. (2012). Prior to analysis, the carbon coating was removed from sample areas of $14 \times 14 \mu \mathrm{m}^{2}$ in size by sputtering with a high current primary beam ( $20 \mathrm{pA})$. Oxygen and subsequent $\mathrm{C}, \mathrm{N}$ analyses were then performed within these areas with the "chained analysis" setup, i.e., after defining a starting position, measurements are carried out automatically, with the sample stage moving in a predefined pattern to subsequent areas. To identify O-anomalous signatures, secondary ion images of ${ }^{16} \mathrm{O}^{-},{ }^{17} \mathrm{O}^{-},{ }^{18} \mathrm{O}^{-},{ }^{28} \mathrm{Si}^{-}$, and ${ }^{27} \mathrm{Al}^{16} \mathrm{O}^{-}$were acquired in multi-collection mode. 
Each individual measurement consisted of 4-5 image planes of 11 minutes integration time each over $10 \times 10 \mu \mathrm{m}^{2}$ areas $(256 \times 256$ pixels $)$. Presolar silicate and oxide grains were identified by their anomalous $\mathrm{O}$-isotopic composition, and detection of ${ }^{28} \mathrm{Si}^{-}$and ${ }^{27} \mathrm{Al}^{16} \mathrm{O}^{-}$ allowed an initial distinction between silicates and Al-rich oxides. A grain was considered presolar if its O-isotopic composition differs by at least $4 \sigma$ from that of the surrounding matrix. The measured O-isotopic compositions of presolar grains were normalized to O-rich material in the surrounding matrix, which was assumed to have solar system isotopic ratios. Presolar grain abundances were calculated by dividing the visible area of all grains in certain area (determined either from the isotopic ratio images or, if available, from high-resolution SEM images) by the total scanned area. Additionally, ${ }^{12} \mathrm{C}^{-},{ }^{13} \mathrm{C}^{-},{ }^{12} \mathrm{C}^{14} \mathrm{~N}^{-},{ }^{12} \mathrm{C}^{15} \mathrm{~N}^{-}$, and ${ }^{28} \mathrm{Si}^{-}$ were measured in multi-collection mode to determine the $\mathrm{N}$-isotopic composition of organic material in several FGR and ICM regions. During these measurements, 4-5 image planes of 11 minutes integration time each were acquired over $10 \times 10 \mu \mathrm{m}^{2}$-sized areas $(256 \times 256$ pixels $)$. Nitrogen isotopic ratios were normalized to those measured on a synthetic N-doped SiCstandard. All obtained data were processed with in-house software developed at the MPIC.

\subsection{Auger Electron Spectroscopy (AES)}

The elemental compositions of presolar silicate grains in the two CR2 chondrites GRA 95229 and EET 92161 were analyzed with the PHI 700 Auger Nanoprobe at Washington University in St. Louis, using established procedures for presolar silicate grains (STADERMANN et al. 2009). Briefly, Auger electron energy spectra are obtained with a $10 \mathrm{kV} 0.25 \mathrm{nA}$ primary electron beam, which is rastered over the grains of interest, allowing to determine the elemental compositions with a lateral resolution of $10-20 \mathrm{~nm}$. Multiple spectral scans of a given grain are added together to obtain a single Auger spectrum, which is acquired in $1 \mathrm{eV}$ steps and then subjected to a 7-point Savitsky-Golay smoothing and differentiation routine 
170 prior to peak identification and quantification. Sensitivity factors for the major rock-forming

171 elements $(\mathrm{O}, \mathrm{Si}, \mathrm{Fe}, \mathrm{Mg}, \mathrm{Ca}$, and $\mathrm{Al})$ were determined from olivine and pyroxene standards of 172 various compositions.

\subsection{SEM-FIB/TEM analysis of FGR material}

175 A total of 4 lamellae from 3 FGRs were prepared with the "focused ion beam" (FIB) 176 technique (e.g., WIRTH 2004; ZEGA et al. 2007). With the FEI Nova 600 Nanolab Dual-Beam

177 FIB Workstation at the Max Planck Institute for Polymer Research (MPIP) in Mainz, thin $178(\sim 100-150 \mathrm{~nm})$ slices for subsequent Transmission electron microscopy (TEM) analyses were 179 produced. TEM analyses were performed on a Zeiss Libra 200FE (200 kV) at the Institute for 180 Mineralogy at the University of Münster for bright field (BF) imaging, selected area electron 181 diffraction (SAED), EDS measurements, and scanning TEM-high angle annular dark field 182 (HAADF) images. X-ray counts were collected for $\sim 100 \mathrm{~s}$ using an ultra-thin window Noran spectrometer, to obtain a relative counting statistical error of $<10 \%$. The spectra were quantified using standard background corrections and Cliff-Lorimer thin-film criteria, the detection limit for major elements with these settings is $\sim 0.5$ at. $\%$. 


\section{RESULTS AND DISCUSSION}

\subsection{Identification of presolar silicate and oxide grains in rims and matrix.}

190

191

192

193

194

195

196

197

198

199

200

201

202

203

204

205

206

207

208

209

We investigated a total of 9 fine-grained chondrule rims in 5 moderately to extensively altered CR2 chondrites (1 in EET 92161, 2 in GRA 95229, 2 in MIL 07525, 1 in NWA 801, 3 in Renazzo), together with selected interchondrule matrix regions in each meteorite by NanoSIMS ion imaging. A total area of $57,600 \mu \mathrm{m}^{2}$ of fine-grained material was scanned $\left(33,300 \mu \mathrm{m}^{2}\right.$ of FGRs, $1,100 \mu \mathrm{m}^{2}$ of fine-grained lithic clast material, and $23,200 \mu \mathrm{m}^{2}$ of ICM), and 43 presolar O-rich grains were identified (39 silicates and 4 Al-rich oxides, Table 2). The majority (33) of these grains (30 silicates, 3 Al-oxides) are located in fine-grained rims (corresponding to a grain density of $990 \pm 170 \mathrm{~mm}^{-2}$ in the FGRs). One presolar silicate was found in a fine-grained lithic clast, while 9 O-anomalous grains ( 8 silicates, 1 Al-oxide) were found in the "classical" interchondrule matrix, representing a grain density of $390 \pm 130$ $\mathrm{mm}^{-2}$ in the ICM (Table 2, Figure 2).

In addition to the moderately to extensively altered CR2 chondrites, we also studied one FGR $\left(5,600 \mu \mathrm{m}^{2}\right)$ and a similar-sized ICM area $\left(5,900 \mu \mathrm{m}^{2}\right)$ in the minimally altered CR2 chondrite MET 00426, together with two FGRs in the C-ungrouped chondrite Acfer $094\left(3,550 \mu \mathrm{m}^{2}\right)$. The rim areas in Acfer 094 contained 9 presolar silicates (according to a grain density of $2500 \pm 850 \mathrm{~mm}^{-2}$ ), while the MET FGR and ICM contained 6 presolar O-anomalous grains (five silicates and one Al-rich oxide grain) and 19 presolar silicate grains, respectively (Table 3, Figure 2), corresponding to grain densities of $1070 \pm 440 \mathrm{~mm}^{-2}$ and $3220 \pm 740 \mathrm{~mm}^{-2}$.

\subsection{Presolar grain abundances.}


The 43 O-anomalous grains in the moderately to extensively altered CR2 chondrites studied here represent an overall presolar grain abundance of $45 \pm 7 \mathrm{ppm}(42 \pm 7 \mathrm{ppm}$ for silicates and $3(+2 /-1)$ ppm for oxides). All errors are $1 \sigma$ and were based on the confidence limits for small numbers according to GEHRELS (1986). The majority of the presolar silicate and oxide grains were found in FGRs, with $\mathrm{O}$-anomalous grain abundances that are lower by about a factor of 3 in the ICM compared to FGRs, when we calculate the "total" abundances for all studied rims and all matrix areas in all moderately to extensively altered CR2s (for the reason of improved counting statistics, see Figure 3).

If we assume that the precursor material in the $\mathrm{CR}$ formation reservoir in the solar nebula was well-mixed, the distribution of presolar grains should have been more or less uniform. DAVIDSON et al. (2014) investigated the abundances of presolar SiC in chondritic meteorites and found no significant variations. However, $\mathrm{SiC}$ and oxygen-rich dust particles condense

first-order assumption, no significant preferential destruction of silicate stardust occurred, especially in the FGRs, emphasizing their primitive nature, with the exception of Renazzo. The lower silicate stardust concentrations in the Renazzo rims could be indicative of more severe aqueous alteration that also reduced the presolar silicate concentration in the FGRs. This observation is also supported by an average presolar O-anomalous grain abundance of 14 (+11/-5) ppm for FGRs in the CM chondrites (calculated from data given in LEITNER et al. 2013), which generally experienced more severe aqueous alteration than the CR chondrites (e.g., BREARLEY 2006). We observe a predominance of presolar silicates over oxides for all samples, independent from the degree of alteration experienced by the host meteorite. Therefore, following the assumption that presolar silicates were preferentially destroyed by 
aqueous alteration, while refractory oxides were more resistant (FLOSS \& STADERMANN 2009; LEITNER et al. 2012), alteration in the CR2 chondrites was not severe enough to cause a significant difference in the presolar silicate/oxide ratios in comparison to the most pristine meteorites. Due to the overall low presolar oxide abundances and the resulting large statistical errors, no significant difference in the silicate/oxide ratios of FGRs and ICM could be observed.

For the CR chondrite MET 00426, we observe that the ICM contains $\sim 5$ times more Oanomalous grains than the FGR (Figure 3). For Acfer 094, the large error for the abundance of presolar grains in FGR material precludes a meaningful comparison. Similar trends are observed for rims and matrix in several $\mathrm{CO} 3.0$ chondrites (Figure 3), with the abundance difference between ICM and FGRs attributed to more extensive aqueous alteration affecting the CO rims (HAENECOUR et al. 2014).

While the average abundances for FGRs and ICM in the minimally altered type 2 and the type 3 chondrites could, in principle, be attributed to more severe aqueous alteration affecting the rims than the interchondrule matrix, this explanation does not hold for the CR2 chondrites. Here, the FGRs cannot have formed by simple (parent body) aqueous alteration of finegrained material in the vicinity of the chondrules, since this should result in even lower grain abundances than those observed in the ICM of the meteorites. Instead, a two-stage alteration model seems more plausible. First, the FGRs formed prior to chondrule incorporation into the parent body. During this period, the rim material experienced compaction to a certain degree, which also led to a reduction of the presolar grain abundances (see also section 3.5.). Subsequent parent body alteration resulted in further reduced abundances in the ICM of the moderately to more severely altered CR2 chondrites, while the potential compaction experienced by the FGRs preserved more presolar grains here. Although the low counting 
FGR and ICM grain abundances for the moderately to more severely altered CR chondrites support this interpretation.

\subsection{Nitrogen isotopic composition, mineralogy, and microstructure of the rim material.}

The FGRs from GRA 95229, EET 92161, and Renazzo also show significant enrichments in ${ }^{15} \mathrm{~N}$ (Table S2, Figure 4). One FGR in GRA 95229 has an average $\delta^{15} \mathrm{~N}$ of $185 \pm 2 \%$, similar to the $\mathrm{N}$-isotopic composition of insoluble organic matter $(\mathrm{IOM})$ in this meteorite $\left(\delta^{15} \mathrm{~N}=\right.$ $153 \pm 7 \%$, AlEXANDER et al. 2007). Isotopic hotspots with $\delta^{15} \mathrm{~N}$ of $500-750 \%$ were also found. A FGR in EET 92161 displays an average $\delta^{15} \mathrm{~N}$ of $55 \pm 7 \%$, significantly lower than the typical ${ }^{15} \mathrm{~N}$-enrichment of CRs, but in the same range that we observe for interchondrule matrix in GRA $95229\left(\delta^{15} \mathrm{~N} 65 \pm 4 \%\right.$ \% . However, individual measurement areas in the EET rim show a wide spread of values, $\sim 0 \%<\delta{ }^{15} \mathrm{~N}<273 \%$. Hotspots with ${ }^{15} \mathrm{~N}$-enrichments of up to $730 \%$ are observed. FGRs in Renazzo have an average $\delta^{15} \mathrm{~N}$ of $\sim 107 \pm 2 \%$; and one ${ }^{15} \mathrm{~N}$-rich hotspot $(730 \pm 40 \%$ ) was detected. The presence of heavy nitrogen-bearing organics gives further evidence for the nebular origin of the rim material, as well as a common origin for the ICM and FGR material. ${ }^{15} \mathrm{~N}$-enriched insoluable organic material is commonly observed in the matrix of CR chondrites (e.g., ALEXANDER et al. 2007). Extensive aqueous or thermal alteration would significantly affect and ultimately equilibrate the isotopic anomaly carried by these materials. The variation of the $\mathrm{N}$-anomaly over individual measurement areas (Figure 4) hints towards heterogeneous aqueous alteration.

We carried out TEM investigations on four FIB-prepared lamellae from presolar grainbearing FGRs (two from GRA 95229, and one each from EET 92161 and MIL 07525, including the two rims containing $\mathrm{N}$-anomalies) to study mineralogical textures. The FGRs in GRA 95229 consist of a complex assemblage of Fe-sulfides, anhydrous Mg-rich silicates, 
nearly unaltered Fe,Ni-metal grains and phyllosilicates (serpentine), all set in a partly amorphous Fe-rich silicate groundmass. Carbonaceous matter is also present as irregular interstitial material throughout the FIB sections, partially as well-preserved nanoglobules (Figure 5). Some fayalitic olivines are recrystallized, indicating alteration, and several Fesulfides display alteration rims. However, the overall texture is very similar to the finegrained material from other primitive carbonaceous chondrites with sulfide-rich and -poor areas and a comparably high porosity, e.g., MET 00426, QUE 99177 (ABREU \& BREARLEY 2010), ALHA 77307 (BREARLEY 1993), or Yamato 791198 (CHIZMADIA \& BREARLEY 2008).

We estimate the fraction of amorphous silicates in the GRA 95229 rim roughly to be $\sim 10-20$ $\%$. The rim material from MIL 07525 contains only a few anhydrous silicates, and even these show signs of alteration. Amorphous silicates make up 1-10 \% of this rim. The FGR in EET 92161 shows signs of more extensive aqueous alteration and contains virtually no amorphous silicates $(<1 \%)$. The presence of such (at least partially) primitive assemblages with hotspots of heavy N, globular organic material and presolar dust in the FGRs supports the idea that FGRs were formed by accretion of dust and ice in the solar nebula on chondrule surfaces prior to parent body formation, as suggested by METZLER et al. (1992). No correlation was observed between the content of amorphous silicates and the presolar grain abundances (Figure 3) of the respective rims.

\subsection{Presolar grain compositions as indicators for secondary processing.}

It has been demonstrated previously that presolar grains can be used as "analytical tools" to assess the degree of alteration experienced by the host material (e.g., FLOSS \& STADERMANN 2009; Leitner et al. 2012; Floss \& StADERMAnN 2012). This is true not only for the abundances and abundance ratios of different presolar grain species, but also for the elemental 
compositions of individual grains, which can give hints about possible aqueous or thermal alteration.

The elemental composition of 19 presolar silicates from GRA 95229 and EET 92161 were investigated by AES (Auger Electron Spectroscopy) from which $(\mathrm{Mg}+\mathrm{Fe}) / \mathrm{Si}$ and $\mathrm{Mg} /(\mathrm{Mg}+\mathrm{Fe})$ ratios were determined (Table 4, Figure 6). All grains are ferromagnesian silicates. The $(\mathrm{Mg}+\mathrm{Fe}) / \mathrm{Si}$ ratios follow a pattern similar to that observed for the presolar silicates in other primitive chondrites (FLOSS \& STADERMANN 2009; VOLLMER et al. 2009a; NGUYEN et al. 2010; ZhaO et al. 2013; Floss \& StADERMAnN 2012; Bose et al. 2010; Bose et al. 2012). Three grains show $(\mathrm{Mg}+\mathrm{Fe}) / \mathrm{Si}$ ratios compatible with a pyroxene-like (Px) composition, four grains have olivine-like $(\mathrm{Ol})(\mathrm{Mg}+\mathrm{Fe}) / \mathrm{Si}$ ratios and six silicates have intermediate compositions, with $(\mathrm{Mg}+\mathrm{Fe}) / \mathrm{Si}$ between $1(\mathrm{Px})$ and $2(\mathrm{Ol})$ (cf. (29)). One grain (GR95_14_13) has a $(\mathrm{Mg}+\mathrm{Fe}) / \mathrm{Si}$ significantly below 1 , and an O/Si-ratio of $\sim 2 . \mathrm{Mg}$ and $\mathrm{Fe}$ are present in the spectrum; therefore, it is not a pure $\mathrm{SiO}_{2}$-grain, but possibly a GEMS-like ("glass with embedded metal and sulfide") grain. Another three grains have Si-poor compositions, and two grains (GR95_13_27, GR95_13_29) display complex compositions, with differing $(\mathrm{Mg}+\mathrm{Fe}) / \mathrm{Si}$ ratios for each of the 2-3 spectra acquired for individual subregions, corresponding to composite structures visible in the SEM (an example for GR95_13_29 is shown in Figure S1). The investigated grains show $\mathrm{Mg} /(\mathrm{Mg}+\mathrm{Fe})$ ratios ranging from 0.2 to 0.8 . This is, on average, more Fe-rich than predicted by current stellar models and astronomical observations (e.g., DEMYK et al. 2000) and has been observed for the presolar silicate populations in the most primitive chondrites like Acfer 094 (VOLLMER et al. 2009a,b; BosE et al. 2010), ALHA 77307 (NGUYEN et al. 2010; BoSE et al. 2012), QUE 99177 (Floss \& StAdERMANn 2009), MET 00426 (Floss \& StADERMANn 2009), and GRV 021710 (ZHAO et al. 2013). The overall compositions of the grains in the FGR and ICM materials studied here show no significant differences from what is observed in chondrites 
showing only very little evidence of aqueous alteration. In addition, we do not observe widespread alteration effects like alteration rims, "haloes", or fuzzy grain boundaries that would be indicative of extensive aqueous alteration (e.g. BREARLEY 2006).

We also did not observe any visible effects of thermal alteration on the presolar silicates. In their study of the C2-ungrouped chondrite Adelaide, FLOSS \& STADERMANN (2012) observed "coarsening" of the matrix in more altered areas, attributed to thermal annealing of the material. Moreover, a large fraction of the studied presolar grains showed compositions that were significantly more Fe-rich than presolar silicates from other primitive chondrites, which the authors also attributed to thermal alteration effects. Clearly, the grains found in this study did not suffer such thermal alteration. Since the FGR-material was accreted onto presumably hot chondrules, this observation indicates a rapid cooling for the accreting chondrules.

\subsection{Implications for rim formation and aqueous alteration of the fine-grained matter.}

For the FGRs investigated in this study, origin and possible formation processes can be further constrained. From the presence of presolar stardust together with the overall composition and texture of the fine-grained host material, rim formation from chondrule debris or by chondrule erosion after incorporation into a parent body can be excluded. Nebular accretion of fine-grained materials unto chondrule surfaces has been discussed by several authors (e.g., BLAND et al. 2011; CIESLA et al. 2003), and recent studies (BEITZ et al. 2013a; BEITZ et al. 2013b) demonstrated experimentally the feasibility of rim accretion under nebular conditions. In such "nebular accretion" scenarios, model-derived and experimentally determined rim porosities are much higher than what is observed in chondritic FGRs. Therefore, additional compaction processes must have taken place either in the nebula or on the respective parent bodies. Compaction of the fine-grained rim material on the parent body 
has been proposed (TRIGO-RODRIGUEZ et al. 2006); however, the fine-grained material itself was believed to have accreted onto the chondrule surface prior to parent body formation. TRIGO-RODRIGUEZ et al. (2006) invoked "collisional compaction processes within parent bodies" to explain the formation of FGRs, but other researchers (BEITZ et al. 2013a,b) did not observe any signs of a density increase for the rims around the chondrule analogues of their experiments, which would have been indicative for the aforementioned parent body compaction processes. It is also not made clear why such internal processes would produce sharply defined boundaries between rims as we observed in the chondritic material. Therefore, we consider this hypothesis as unlikely for the production of FGRs.

Volume filling factors might be enhanced by aqueous alteration, as suggested by TAKAYAMA \& TomeOKA (2012) for rims in the Tagish Lake meteorite. This could, in theory, explain the fact that the observed porosities in meteoritic FGRs are higher than in theoretical predictions and experimental results. However, those rims are extensively altered and consist primarily of phyllosilicates, while the FGRs in the present study represent a relatively primitive mixture of anhydrous and altered phases showing no evidence for extensive aqueous modification.

Impacts have been suggested as relevant parent-body processes for the compaction of finegrained material in the vicinity of chondrules (BLAND et al. 2011); however, these processes are not considered as fundamental for the formation of FGRs in chondrites (BLAND et al. 2011). Low-energy impacts are invoked as mechanism for compaction and lithification (BLAND et al. 2012, 2014), thus preserving accretionary fabrics as observed in Allende (e.g., (BLAND et al. 2011). Evidence for multiple low-intensity impacts responsible for the compaction and formation of petrofabrics has been found in CM chondrites (LINDGREN et al. 2014). Such low-pressure shock events would be accompanied by fluid volatilization (BLAND et al. 2012). Recent work by BLAND et al. (2014) demonstrated that low-energy impacts, which would cause no shock signatures in compact meteoritic components like chondrules, 
can cause significant temperature increases (exceeding $1000 \mathrm{~K}$ in localized spots) in the adjacent fine-grained porous material. Such heating events would be short $(<10$ s), with the chondrules acting as heat sinks. These effects could explain (partially) our observations for the moderately to more severely altered CR2 chondrites: In the chondrite formation region in the early solar nebula, a fraction of the chondrules accreted fine-grained dust rims. These rims were (partially) disrupted and compacted by multiple collisions occurring during parent body formation. After FGR accretion, the material was aqueously altered by shock-released water from accreted (water) ice grains, which lead to a reduced presolar silicate abundance compared to the fine-grained precursor material, depending on the shock energy and the amount of accreted ice. Isotopic homogenization of presolar grains on timescales of $\sim 0.1-10 \mathrm{~s}$ can be expected (BLAND et al. 2012), further reducing the concentration of presolar material in the FGRs. From this reservoir of fine-grained dust, rimmed and rimless chondrules, as well as other larger constituents, chondritic parent bodies accreted. During this formation process, the fine-grained component experienced impact-induced shock heating as outlined by BLAND et al. (2014), which further modified its presolar grain content.

BLAND et al. (2011) find evidence from fabric analysis of matrix and fine-grained rim material in the Allende meteorite (CV3) for nebular accretion and compaction of the rim structure. They report in their study the absence of any strain to the rock fabric of a FGR, resulting in a spherically symmetric stress field. The absence of strain shadows in the rock fabric, together with the observation of the juxtaposition of a uniaxial and a spherically symmetric stress field contradict models which suggest parent body compaction processes (e.g., TOMEOKA \& TANimura 2000; Trigo-RodrigueZ et al. 2006). The latter models would require multiple impacts on the parent body, which would not allow the preservation of such spherically symmetric stress fields. Moreover, multiple impacts would preclude the recording 

(2011).

408

Several studies (BLum \& SCHRÄPleR 2004; ORMEL et al. 2008; DOMINIK \& TIELENS 1997) predicted high initial FGR porosities after accretion in the nebula, which is in good accordance with the estimates by BLAND et al. (2011) as well as the observations by BEITZ et al. (2013a,b). Compaction would then occur by combinations of chondrule-dust-interactions and low-intensity shocks. Impact-induced shock heating, even at low collision velocities $(\mathrm{v} \sim 0.75-2 \mathrm{~km} / \mathrm{s})$, could result in bulk temperatures of $\sim 400-700 \mathrm{~K}$ in the fine-grained material (with localized spots experiencing $\mathrm{T}($ peak) $>1,000 \mathrm{~K}$ ), but would not be recorded in the compact chondrules, which would act as heat sinks. This would create heterogeneous structures on the sub-mm scale, as are observed in chondritic meteorites, and allow for fast cooling (<10 s) and lithification of the fine-grained material (BLAND et al. 2014).

We envision the processes responsible for the observed differences in the presolar grain abundances as following:

For pristine or minimally altered carbonaceous chondrites, the very primitive ICM displays high presolar grain abundances in the range of 150 to 250 ppm (FLOSS \& STADERMANN 2009; VolLmer et al. 2009a, HAENECOUR et al. 2014), while FGRs can display lower abundances (Figure 3).

For moderately altered carbonaceous chondrites, like the majority of CR2 chondrites investigated in this study, aqueous parent body alteration has decreased the presolar silicate abundances in the respective ICM, while the FGRs were "protected" to a certain degree by their compacted nature.

In more severely altered chondrites, like Renazzo in this study, and the CM chondrites studied by LEITNER et al. (2013), presolar silicate abundances in ICM and FGRs were further reduced 
by more extensive aqueous alteration. Due to low presolar grain statistics, it was not possible to identify differences between the abundances in FGRs and ICM for individual meteorites in many cases. Nevertheless, when we calculate the respective abundances for the total investigated ICM and FGR areas, the differences become visible (Figure 3).

\section{CONCLUSIONS}

Presolar silicate and oxide grains are present in several fine-grained chondrule rims of carbonaceous chondrites which experienced varying degrees of aqueous alteration, ranging from nearly pristine/minimally altered to more severely altered. Due to limited statistics, we observe differences of presolar O-rich grain abundances between FGRs and ICM for individual meteorites only with 1-sigma significance in most of the cases (Figure 3, Table S1). However, the "total" or combined grain abundance for the FGRs in all investigated samples and the respective total abundance for the ICM, differ by a factor of 3 for the moderately and more severely altered CR chondrites. For the minimally altered CR chondrite MET 00426, we observed higher abundances in the interchondrule matrices than in the finegrained rims, and for the ungrouped carbonaceous chondrite Acfer 094, the values for ICM and FGR overlap within the error limits.

The elemental compositions of presolar silicate grains in the moderately altered CR2 chondrites GRA 95229 and EET 92161 do not differ significantly from those of presolar silicates and oxides found in the most primitive carbonaceous chondrites, i.e., no signs for significantly higher degrees of alteration for the grains were observed. The fine-grained rims contain organic material with ${ }^{15} \mathrm{~N}$-excesses similar to what is typically observed in the matrix of $\mathrm{CR}$ chondrites. The $\mathrm{N}$-anomalies are inherited from the protosolar nebula (e.g., ALEXANDER et al. 2007; BUSEMANN et al. 2006), and their presence in the FGRs further 
indicates a nebular origin of the rim material. Obviously, the material did not experience any alteration that would have been capable of diluting or even homogenize the anomalous isotopic signatures. TEM investigations revealed that the FGR material in the vicinity of preserved presolar grains consists of a heterogeneous mixture of pristine nebular material and components showing various degrees of aqueous alteration.

In summary, these observations indicate a nebular origin of the fine-grained material comprising the FGRs studied here. The observed differences between the total presolar silicate and oxide abundances in the fine-grained rims and the interchondrule matrix of the moderately to more severely altered CR2 chondrites and the minimally altered carbonaceous chondrites of this study imply different alteration paths for the fine-grained rims and the $\mathrm{C} 2$ and C3-matrix. This indicates a nebular origin of the FGRs, and incorporation by accretion for the rimmed chondrules. Furthermore, our findings clearly show that fine-grained chondrule rims like the ones investigated in this study cannot have formed by chondrule erosion or regolith gardening processes on the respective parent bodies. However, one has to keep in mind that chondritic meteorites contain various types of chondrule rims. Thus, different formation scenarios might very well apply for other rims that have been labelled "finegrained" despite a clearly different composition or structure (e.g., TAKAYAMA \& TOMEOKA 2012), and a universal rim formation process seems rather unlikely. 


\section{REFERENCES}

Alexander, C. M. O’D., Fogel, M., Yabuta, H., and Cody, G. D. 2007. The origin and evolution of chondrites recorded in the elemental and isotopic compositions of their macromolecular organic matter. Geochim. Cosmochim. Acta, 71, 4380-4403.

Alexander, C. M. O’D., Howard, K. T., Bowden, R., and Fogel, M. L. 2013. The classification of $\mathrm{CM}$ and $\mathrm{CR}$ chondrites using bulk $\mathrm{H}, \mathrm{C}$ and $\mathrm{N}$ abundances and isotopic compositions. Geochim. Cosmochim. Acta, 123, 244-260.

Allen, J. S., Nozette, S., and Wilkening, L. L. 1980. A study of chondrule rims and chondrule irradiation records in unequilibrated ordinary chondrites. Geochim. Cosmochim. Acta, $44,1161-1175$.

Beitz, E., Güttler, C., Nakamura, A. M., Tsuchiyama, A., and Blum, J. 2013a. Experiments on the consolidation of chondrites and the formation of dense rims around chondrules. Icarus, 225, 558-569.

Beitz, E., Blum, J., Mathieu, R., Pack, A., and Hezel, D. 2013b. Experimental investigation of the nebular formation of chondrule rims and the formation of chondrite parent bodies. Geochim. Cosmochim. Acta, 116, 41-51.

Bland, P. A., Stadermann, F. J., Floss, C., Rost, D., Vicenzi, E. P., Kearsley, A. T., and Benedix, G. K. 2007. A cornucopia of presolar and early solar system materials at the micrometer size range in primitive chondrite matrix. Meteorit. Planet. Sci., 42, $1417-$ 1427.

Bland, P. A., Howard, L. E., Prior, D. J., Wheeler, J., Hough, R. M., and Dyl, K. A. 2011. Earliest rock fabric formed in the Solar System preserved in a chondrule rim. Nat. Geosci., 4, 244-247. 
Bland, P. A., Muxworthy, A. R., Collins, G. S., Moore, J., Davison, T. M., Prior, D. J., Wheeler, J., Ciesla, F. J., and Dyl, K. A. 2012. Effect of low intensity impacts on chondrite matrix. In Lunar Planet. Sci. Conf. 43, ed. S. J. Mackwell (Houston, TX: LPI), 2005.

Bland, P.A., Collins, G.S., Davison, T.M., Abreu, N.M., Ciesla, F.J., Muxworthy, A.R., and Moore, J. 2014. Pressure-temperature evolution of primordial solar system solids during impact-induced compaction. Nat. Communications, doi: 10.1038/ncomms6451.

Blum, J. and Schräpler, R. 2004. Structure and Mechanical Properties of High-Porosity Macroscopic Agglomerates Formed by Random Ballistic Deposition. Phys. Rev. Lett., $93,115503-1$.

Bose, M., Floss, C., and Stadermann, F. J. 2010. An Investigation into the Origin of Fe-rich Presolar Silicates in Acfer 094. Astrophys. J., 714, 1624-1636.

Bose, M., Floss, C., Stadermann, F. J., Stroud, R. M., and Speck, A. K. 2012. Circumstellar and interstellar material in the CO3 chondrite ALHA77307: An isotopic and elemental investigation. Geochim. Cosmochim. Acta, 93, 77-101.

Brearley, A. J. 2006. The Action of Water. In Meteorites and the Early Solar System II, D. S. Lauretta and H. Y. McSween Jr. (eds.), University of Arizona Press, Tucson, 943 pp., p.584-624

Busemann, H., Young, A. F., Alexander, C. M. O’D., Hoppe, P., Mukhopadhyay, S., and Nittler, L. R. 2006. Interstellar Chemistry Recorded in Organic Matter from Primitive Meteorites. Science, 312, 727-730. 
Choi, B.-G., Huss, G. R., Wasserburg, G. J., and Gallino, R. 1998. Presolar Corundum and Spinel in Ordinary Chondrites: Origins from AGB Stars and a Supernova. Science, 282,

\section{$1284-1289$.}

Ciesla, F. J., Lauretta, D. S., Cohen, B. A., and Hood, L. L. 2003. A Nebular Origin for Chondritic Fine-Grained Phyllosilicates. Science, 299, 549.

Chizmadia, L. J. and Brearley A. J. 2008. Mineralogy, aqueous alteration, and primitive textural characteristics of fine-grained rims in the Y-791198 CM2 carbonaceous chondrite: TEM observations and comparison to ALHA81002. Geochim. Cosmochim. Acta, 72, 602-625.

Davidson, J. 2009. NanoSIMS and Beyond: Presolar grains in primitive materials and Solar System formation. Ph.D. dissertation, Open University, Milton Keynes, UK.

Davidson, J., Busemann, H., and Franchi, I. A. 2012. Presolar grain inventory of a finegrained rim in the CO3.0 ALHA 77307. Meteorit. Planet. Sci. Suppl., Abstract \#5269.

Davidson, J., Busemann, H., Nittler, L. R., Alexander, C. M. O’D. Orthous-Daunay, F.-R. Franchi, I. A, and Hoppe, P. 2014. Abundances of presolar silicon carbide grains in primitive meteorites determined by NanoSIMS. Geochim. Cosmochim. Acta, 139, 248 266.

Davidson, J., Alexander, C. M. O’D., Schrader, D. L., Nittler, L. R., and Bowden, R.. 2015. Miller Range 090657: A very pristine Renazzo-like (CR) Carbonaceous Chondrite. In Lunar Planet Sci. Conf. 44, ed. S. J. Mackwell (Houston, TX: LPI).

Demyk, K., Dartois, E., Wiesemeyer, H., Jones, A. P., and d'Hendecourt, L. 2000. Structure and chemical composition of the silicate dust around OH/IR stars. Astron. Astrophys., 364, 170-178. 
Dominik, C. and Tielens, A. G. G. M. 1997. The physics of dust coagulation and the structure of dust aggregates in space. Astrophys. J., 480, 647-673.

Floss, C., and Stadermann, F. J. 2005. Presolar (circumstellar and interstellar) phases in Renazzo: The effects of parent body processing. In Lunar Planet Sci. Conf. 36, ed. S. J. Mackwell (Houston, TX: LPI), 1390.

Floss, C., and Stadermann, F. J. 2009. Auger Nanoprobe analysis of presolar ferromagnesian silicate grains from primitive CR chondrites QUE 99177 and MET 00426 Geochim. Cosmochim. Acta, 73, 2415-2440.

Floss, C., and Stadermann, F. J. 2012. Presolar silicate and oxide abundances and compositions in the ungrouped carbonaceous chondrite Adelaide and the $\mathrm{K}$ chondrite Kakangari: The effects of secondary processing. Meteorit. Planet. Sci., 47, 992-1009.

Gehrels, N. 1986. Confidence Limits for small Numbers of Events in Astrophysical Data. Astrophys. J., 303, 336-346.

Haenecour, P. and Floss, C. 2012. Stardust in Fine-Grained Chondrule Rims and Matrix in LaPaz 031117: Insights into the Conditions of Dust Accretion in the Solar Nebula. In Lunar Planet. Sci. Conf. 43, ed. S. J. Mackwell (Houston, TX: LPI), 1659.

Haenecour, P., Floss, C., Joliff, B. L., Zega, T. J., Bose, M., and Carpenter, P. 2014. Presolar silicates as tracers of the formation of fine-grained chondrule rims in $\mathrm{CO} 3$ chondrites. In Lunar Planet. Sci. Conf. 45, ed. S. J. Mackwell (Houston, TX: LPI), 1316.

Harju, E. R., Rubin, A. E., Ahn, I., Choi, B.-G., Ziegler, K., and Wasson, J. T. 2014. Progressive aqueous alteration of CR carbonaceous chondrites. Geochim. Cosmochim. Acta, 139, 267-292. 
Howard, K. T., Alexander, C.M.O’D., Schrader, D.L., and Dyl, K.A. 2015. Modal mineralogy of CM2 chondrites by X-ray diffraction (PSD-XRD). Part 1: Total phyllosilicate abundance and the degree of aqueous alteration. Geochim. Cosmochim. Acta, 149, 206222.

Huss, G. R., Meshik, A. P., Smith, J. B., and Hohenberg, C. M. 2003. Presolar diamond, silicon carbide, and graphite in carbonaceous chondrites: Implications for thermal processing in the solar nebula. Geochim. Cosmochim. Acta, 67, 4823-4848.

Krot, A. N., Meibom, A., Weisberg, M. K., and Keil, K. 2002. The CR chondrite clan: Implications for early solar system processes. Meteorit. Planet. Sci., 37, 1451-1490.

Le Guillou, C. and Brearley, A. 2014. Relationships between organics, water and early stages of aqueous alteration in the pristine CR3.0 chondrite MET 00426. Geochim. Cosmochim. Acta, 131, 344-367.

Leitner, J., Vollmer, C., Hoppe, P., and Zipfel, J. 2012. Characterization of Presolar Material in the CR Chondrite Northwest Africa 852. Astrophys. J. 745, 38-53.

Leitner, J., Metzler, K., Vollmer, C., and Hoppe, P. 2013. Search for Presolar Grains in Finegrained Chondrule Rims: First Results From CM Chondrites and Acfer 094. In Lunar Planet Sci. Conf. 44, ed. S. J. Mackwell (Houston, TX: LPI), 2273.

Lindgren, P., Hanna, R.D., Dobson, K.J., Tomkinson, T., and Lee, M.R. 2014. The paradox between low shock-stage and evidence for compaction in CM carbonaceous chondrites explained by multiple low-intensity impacts. Geochim. Cosmochim. Acta , 148, 159178.

Metzler, K., Bischoff, A., and Stöffler, D. 1992. Accretionary dust mantles in CM chondrites: Evidence for solar nebula processes. Geochim. Cosmochim. Acta, 56, 2873-2897. 
Nagashima, K., Krot, A. N., and Yurimoto, H. 2004. Stardust silicates from primitive meteorites. Nature, 428, 921-924.

Newton, J., Bischoff, A., Arden, J. W., Franchi, I. A., Geiger, T., Greshake, A. and Pillinger, C. T. 1995. Acfer 094, a uniquely primitive carbonaceous chondrite from the Sahara. Meteoritics, 30, 47-56.

Nguyen, A., Stadermann, F. J., Zinner, E., Stroud, R. M., Alexander, C. M. O. D., and Nittler, L. R. 2007. Characterization of presolar silicate and oxide grains in primitive carbonaceous chondrites. Astrophys. J., 656, 1223-1240.

Nguyen, A. N., Nittler, L. R., Stadermann, F. J., Stroud, R. M., and Alexander, C. M. O’D. 2010. Coordinated analyses of presolar grains in the Allan Hills 77307 and Queen Elizabeth Range 99177 meteorites. Astrophys. J., 719, 166-189.

Nittler, L. R., Alexander, C.M.O’D., Gao, X., Walker, R. M., and Zinner, E. 1997. Stellar sapphires: The properties and origins of presolar $\mathrm{Al}_{2} \mathrm{O}_{3}$ in meteorites. Astrophys. J., 483, 475-495.

Nittler, L. R., Alexander, C.M.O’D., Gallino, R., Hoppe, P., Nguyen, A. N., Stadermann, F. J., and Zinner, E. 2008. Aluminum-, Calcium- and Titanium-rich oxide stardust in Ordinary chondrite meteorites. Astrophys. J., 682, 1450-1478.

Nittler, L. R., Alexander, C. M. O'D., and Stroud, R. M. .2013. High Abundance of Presolar Materials in CO3 Chondrite Dominion Range 08006. In Lunar Planet Sci. Conf. 44, ed. S. J. Mackwell (Houston, TX: LPI), \#2367. 
Ormel, C. W., Cuzzi, J. N., and Tielens, A. G. G. M. 2008. Co-accretion of chondrules and dust in the Solar nebula. Astrophys. J., 679, 1588-1610.

Schrader, D. L., Davidson, J., Greenwood, R. C., Franchi, I. A., and Gibson, J. M. 2014. A water-ice rich minor body from the early Solar System: The CR chondrite parent asteroid. Earth Planet. Sci. Lett., 407, 48-60.

Scott, E. R. D., Keil, K. and Stöffler, D. 1992. Shock metamorphism of carbonaceous chondrites. Geochim. Cosmochim. Acta, 56, 4281-4293.

Sears, D. W. G., Benoit, P. H., and Jie, L. 1993. Two chondrule groups each with distinctive rims in Murchison recognized by cathodoluminescence. Meteoritics, 28, 669-675.

Stadermann, F. J., Floss, C., Bose, M., and Lea, A. S. 2009. The use of Auger spectroscopy for the in situ elemental characterization of sub-micrometer presolar grains. Meteorit. Planet. Sci., 44, 1033-1049.

Takayama, A. and Tomeoka, K. 2012. Fine-grained rims surrounding chondrules in the Tagish Lake carbonaceous chondrite: Verification of their formation through parent-body processes. Geochim. Cosmochim. Acta, 98, 1-18.

Tomeoka, K. and Tanimura, I. 2000. Phyllosilicate-rich chondrule rims in the Vigarano CV3 chondrite: Evidence for parent-body processes. Geochim. Cosmochim. Acta, 64, 19711988.

Trigo-Rodriguez, J. M., Rubin, A. E., and Wasson, J. T. 2006. Non-nebular origin of dark mantles around chondrules and inclusions in CM chondrites. Geochim. Cosmochim. Acta, 70, 1271-1290. 
Vollmer, C., Hoppe, P., Stadermann, F. J., Floss, C., and Brenker, F. 2009a. NanoSIMS analysis and Auger electron spectroscopy of silicate and oxide stardust from the carbonaceous chondrite Acfer 094. Geochim. Cosmochim. Acta, 73, 7127-7149.

Vollmer, C., Brenker, F., Hoppe, P., and Stroud, R. M. 2009b. Direct laboratory analysis of silicate stardust from red giant stars. Astrophys. J., 700, 774-782.

Wirth, R. 2004. Focused Ion Beam (FIB): a novel technology for advanced application of micro- and nanoanalysis in geosciences and applied mineralogy. Eur. J. Mineral., 16, 863-876.

Zega,T. J., Nittler, L. R., Busemann, H, Hoppe, P., and Stroud, R. M. 2007. Coordinated isotopic and mineralogic analyses of planetary materials enabled by in situ lift-out with a focused ion beam scanning electron microscope. Meteorit. Planet. Sci., 42, 1373-1386.

Zhao, X., Floss, C., Lin, Y., and Bose, M. 2013. Stardust investigation into the CR chondrite Grove Mountain 021710. Astrophys. J., 769, 49.

Zinner, E. 2014. Presolar Grains. In Meteorites and Cosmochemical Processes, ed. A. M. Davis (Amsterdam: Elsevier), 181-213. R. S. 2003. Presolar spinel grains from the Murray and Murchison carbonaceous chondrites. Geochim. Cosmochim. Acta, 67, 5083-5095. 


\section{ACKNOWLEDGEMENTS}

648 We thank Elmar Gröner for technical support on the NanoSIMS, Joachim Huth and Antje 649 Sorowka for help with the SEM analyses, Dimitri Kuzmin for support on the Electron 650 Microprobe, and Maren Müller and Michael Kappl from the Max Planck Institute for Polymer 651 Research in Mainz, Germany, for preparation of the FIB lamellae analyzed in this study. J.L., 652 P.H., J.Z. and C.V. acknowledge support by DFG through SPP 1385: The first ten million 653 years of the solar system - a planetary materials approach (grants HO 2163/1-1\&2, LE $6543279 / 1-1$, VO 1816/1-1). The work at Washington University in St. Louis is funded by NASA 655 grant NNX14AG25G (C.F.). US Antarctic meteorite samples are recovered by the Antarctic 656 Search for Meteorites (ANSMET) program which has been funded by NSF and NASA, and

657 characterized and curated by the Department of Mineral Sciences of the Smithsonian 658 Institution and Astromaterials Curation Office at NASA Johnson Space Center. We 659 acknowledge the constructive and careful reviews by three anonymous reviewers.

660

\section{AUTHOR CONTRIBUTIONS}

662 J.L., P.H. and J.Z. designed the project; J.L., C.V. and C.F. performed the experiments; J.L., 663 C.V. and C.F. collected and analyzed the data; J.L. wrote the manuscript; P.H., C.V. and C.F. 664 edited the manuscript. 
666 Table 1. Samples and sizes of analyzed areas investigated in this study.

\begin{tabular}{|c|c|c|c|c|c|}
\hline Meteorite & Source & Sample no. & $\begin{array}{l}\text { FGR area } \\
\left.\left(\mu m^{2}\right)^{2}\right) / \text { no. of } \\
\text { studied FGRs }\end{array}$ & $\begin{array}{l}\text { ICM area } \\
\left(\mu \mathrm{m}^{2}\right)\end{array}$ & $\begin{array}{l}\text { Shock } \\
\text { stage }\end{array}$ \\
\hline Acfer 094 (C3-ungr.) & NHM Vienna & M5928 & $3,500 / 2$ & - & $\mathrm{S} 1^{\mathrm{a}}$ \\
\hline EET 92161 (CR2) & NASA JSC & 17 & $6,500 / 1$ & 4,600 & \\
\hline GRA 95229 (CR2) & NASA JSC & 97 & $7,200 / 2$ & 5,700 & $\mathrm{~S} 1^{\mathrm{b}}$ \\
\hline MET 00426 (CR3) & NASA JSC & 13 & $5,600 / 1$ & 5,900 & $\mathrm{~S} 2^{\mathrm{b}}$ \\
\hline MIL 07525 (CR2) & NASA JSC & 09 & $4,800 / 2$ & 4,600 & $\mathrm{~S} 2^{\mathrm{b}}$ \\
\hline NWA 801 (CR2) & NHM London & P11648 & $2,600 / 1$ & 1,800 & \\
\hline Renazzo (CR2) & NHM Vienna & A437/L3812 & $12,200 / 3$ & 6,500 & $\mathrm{~S} 1-\mathrm{S} 3^{\mathrm{c}}$ \\
\hline \multicolumn{6}{|l|}{ a NEWTON et al. 1995} \\
\hline \multicolumn{6}{|l|}{${ }^{\mathrm{b}}$ HARJU et al. 2014} \\
\hline \multicolumn{6}{|l|}{${ }^{\mathrm{c}}$ SCOTT et al. 1992} \\
\hline & & & & & \\
\hline
\end{tabular}


671 Table 2. Presolar silicates and oxides identified in fine-grained chondrule rims,

672 interchondrule matrix, and one lithic matrix clast in the CR2 chondrites of this study. "Gr."

673 denotes the group of the respective grain (NITTLER et al. 1997, 2008); "Min." gives the

674 mineralogy of the grain (sil.: silicate, ox.: (Al-rich) oxide, com.: complex grain with oxide

675 core and silicate rim). Grain sizes in italics were determined from ion images, all other were

676 derived by SEM.

\begin{tabular}{|c|c|c|c|c|c|c|c|c|c|c|c|}
\hline \multirow{2}{*}{$\begin{array}{l}\text { Meteorite } \\
\text { GRA 95229 }\end{array}$} & \multirow{2}{*}{$\begin{array}{l}\text { Loc. } \\
\text { clast }\end{array}$} & \multirow{2}{*}{$\begin{array}{l}\text { Grain } \\
\text { GR95_04_16 }\end{array}$} & \multirow{2}{*}{$\begin{array}{c}\begin{array}{c}\text { Size } \\
(\mathrm{nm})\end{array} \\
490 \times 310\end{array}$} & \multicolumn{3}{|c|}{${ }^{17} \mathrm{O} /{ }^{16} \mathrm{O}\left(\times 10^{-4}\right)$} & \multicolumn{3}{|c|}{${ }^{18} \mathrm{O} /{ }^{16} \mathrm{O}\left(\times 10^{-3}\right)$} & Gr. & Min. \\
\hline & & & & 5.17 & \pm & 0.29 & 1.95 & \pm & 0.06 & 1 & sil. \\
\hline GRA 95229 & ICM & GR95_08_07 & $370 \times 240$ & 7.95 & \pm & 0.76 & 1.80 & \pm & 0.11 & 1 & com. \\
\hline GRA 95229 & FGR & GR95_12_5 & $235 \times 265$ & 6.87 & \pm & 0.40 & 1.88 & \pm & 0.07 & 1 & sil. \\
\hline GRA 95229 & FGR & GR95_12_6 & $250 \times 200$ & 5.16 & \pm & 0.32 & 1.97 & \pm & 0.06 & 1 & sil. \\
\hline GRA 95229 & FGR & GR95_13_6 & $200 \times 145$ & 5.21 & \pm & 0.27 & 2.01 & \pm & 0.05 & 1 & oxide \\
\hline GRA 95229 & FGR & GR95_13_22 & $400 \times 130$ & 6.96 & \pm & 0.61 & 0.33 & \pm & 0.03 & 2 & sil. \\
\hline GRA 95229 & FGR & GR95_13_24 & $245 \times 160$ & 5.11 & \pm & 0.29 & 1.96 & \pm & 0.06 & 1 & sil. \\
\hline GRA 95229 & FGR & GR95_13_27 & $580 \times 360$ & 6.43 & \pm & 0.70 & 0.80 & \pm & 0.08 & 2 & sil. \\
\hline GRA 95229 & FGR & GR95_13_29 & $370 \times 230$ & 62.54 & \pm & 2.51 & 1.96 & \pm & 0.14 & ext. $1^{\mathrm{a}}$ & sil. \\
\hline GRA 95229 & FGR & GR95_14_13 & $400 \times 215$ & 8.29 & \pm & 1.24 & 0.81 & \pm & 0.12 & 2 & sil. \\
\hline Renazzo & FGR & REN_02_20 & $225 \times 250$ & 7.99 & \pm & 0.77 & 1.60 & \pm & 0.11 & 1 & sil. \\
\hline Renazzo & FGR & REN_04_06 & $225 \times 425$ & 5.96 & \pm & 0.35 & 1.82 & \pm & 0.06 & 1 & sil. \\
\hline Renazzo & FGR & REN_08_23 & $220 \times 190$ & 3.75 & \pm & 0.53 & 2.64 & \pm & 0.14 & 4 & sil. \\
\hline EET 92161 & FGR & EET_2B_9 & $205 \times 310$ & 3.62 & \pm & 0.24 & 2.33 & \pm & 0.06 & 4 & sil. \\
\hline EET 92161 & FGR & EET_2B_2 & $495 \times 410$ & 6.43 & \pm & 0.57 & 1.84 & \pm & 0.10 & 1 & sil. \\
\hline EET 92161 & FGR & EET_02A__B_2 & $290 \times 200$ & 6.17 & \pm & 0.80 & 1.29 & \pm & 0.11 & 1 & sil. \\
\hline EET 92161 & FGR & EET_02A_B_3 & $190 \times 200$ & 6.18 & \pm & 0.37 & 2.04 & \pm & 0.07 & 1 & sil. \\
\hline EET 92161 & FGR & EET_02A_B_10 & $430 \times 400$ & 6.09 & \pm & 0.34 & 1.90 & \pm & 0.06 & 1 & sil. \\
\hline EET 92161 & FGR & EET_02A_B_15 & $130 \times 90$ & 10.65 & \pm & 1.40 & 1.92 & \pm & 0.18 & 1 & ox. \\
\hline EET 92161 & FGR & EET_02A_B_31* & $334 \times 334$ & 7.73 & \pm & 0.77 & 1.82 & \pm & 0.12 & 1 & sil. \\
\hline EET 92161 & FGR & EET_03B_5 & $225 \times 310$ & 6.43 & \pm & 0.61 & 2.23 & \pm & 0.11 & 1 & sil. \\
\hline EET 92161 & ICM & EET_4_2 & $175 \times 120$ & 9.47 & \pm & 1.29 & 1.61 & \pm & 0.17 & 1 & sil. \\
\hline EET 92161 & ICM & EET_5B_5 & $145 \times 185$ & 10.62 & \pm & 1.50 & 2.22 & \pm & 0.20 & 1 & sil. \\
\hline EET 92161 & ICM & EET_6_- & $320 \times 220$ & 3.87 & \pm & 1.23 & 11.89 & \pm & 0.69 & 4 & sil. \\
\hline EET 92161 & ICM & EET_6_13 & $175 \times 100$ & 8.44 & \pm & 0.87 & 1.88 & \pm & 0.13 & 1 & sil. \\
\hline EET 92161 & ICM & EET_6_15 & $310 \times 270$ & 9.47 & \pm & 1.79 & 4.70 & \pm & 0.40 & 4 & sil. \\
\hline EET 92161 & ICM & EET_6_14* & $330 \times 330$ & 8.62 & \pm & 0.99 & 1.97 & \pm & 0.15 & 1 & ox. \\
\hline NWA 801 & FGR & NWA801_01B_7_1 & $300 \times 295$ & 24.06 & \pm & 0.16 & 1.62 & \pm & 0.13 & 1 & sil. \\
\hline NWA 801 & FGR & NWA801_01B_7_2 & $190 \times 150$ & 6.23 & \pm & 0.48 & 1.98 & \pm & 0.09 & 1 & sil. \\
\hline NWA 801 & FGR & NWA801_1B_9 & $200 \times 135$ & 6.77 & \pm & 0.71 & 1.81 & \pm & 0.12 & 1 & sil. \\
\hline NWA 801 & FGR & NWA801_01B2_3 & $330 \times 180$ & 5.13 & \pm & 0.28 & 1.75 & \pm & 0.05 & 1 & sil. \\
\hline NWA 801 & FGR & NWA801_01B3_6 & $400 \times 260$ & 5.05 & \pm & 0.27 & 1.96 & \pm & 0.05 & 1 & sil. \\
\hline MIL 07525 & ICM & MIL_R4\#6 & $385 \times 300$ & 14.60 & \pm & 1.22 & 2.01 & \pm & 0.14 & 1 & sil. \\
\hline MIL 07525 & ICM & MIL_R4\#12 & $430 \times 330$ & 7.10 & \pm & 0.51 & 1.67 & \pm & 0.08 & 1 & sil. \\
\hline MIL 07525 & FGR & MIL_R3\#13 & $535 \times 310$ & 19.50 & \pm & 1.18 & 1.86 & \pm & 0.12 & 1 & sil. \\
\hline MIL 07525 & FGR & MIL_R2\#2 & $170 \times 180$ & 10.70 & \pm & 1.19 & 1.80 & \pm & 0.16 & 1 & sil. \\
\hline MIL 07525 & FGR & MIL_R2\#13 & $255 \times 165$ & 13.80 & \pm & 1.50 & 2.30 & \pm & 0.20 & 1 & sil. \\
\hline MIL 07525 & FGR & MIL_R2\#16 & $200 \times 310$ & 4.94 & \pm & 0.61 & 2.72 & \pm & 0.14 & 4 & sil. \\
\hline MIL 07525 & FGR & MIL_R8\#2 & $270 \times 165$ & 10.30 & \pm & 0.97 & 1.60 & \pm & 0.12 & 1 & sil. \\
\hline MIL 07525 & FGR & MIL_R8\#10 & $265 \times 210$ & 11.20 & \pm & 1.08 & 1.81 & \pm & 0.14 & 1 & sil. \\
\hline MIL 07525 & FGR & MIL_R8\#11 & $290 \times 325$ & 7.25 & \pm & 0.73 & 1.62 & \pm & 0.11 & 1 & sil. \\
\hline MIL 07525 & FGR & MIL_R9\#5 & $280 \times 225$ & 5.87 & \pm & 0.37 & 0.90 & \pm & 0.11 & 2 & ox. \\
\hline MIL 07525 & FGR & MIL_R9\#10 & $320 \times 220$ & 6.33 & \pm & 0.88 & 1.38 & \pm & 0.12 & 1 & sil. \\
\hline
\end{tabular}


678 Table 3. Presolar silicates and oxides identified in fine-grained chondrule rims,

679 interchondrule matrix, and one lithic matrix clast in the minimally altered chondrites studied

680 here (MET 00426 (CR), Acfer 094 (C-ungrouped)). "Gr." denotes the group of the respective

681 grain (NITTLER et al. 1997, 2008); "Min." gives the mineralogy of the grain (sil.: silicate, ox.:

682 (Al-rich) oxide). Grain sizes were determined from ion images.

\begin{tabular}{|c|c|c|c|c|c|c|c|c|c|c|c|}
\hline \multirow{2}{*}{$\begin{array}{l}\text { Meteorite } \\
\text { MET } 00426\end{array}$} & \multirow{2}{*}{$\begin{array}{l}\text { Loc. } \\
\text { ICM }\end{array}$} & \multirow{2}{*}{$\begin{array}{l}\text { Grain } \\
\text { MET_01B_10 }\end{array}$} & \multirow{2}{*}{$\begin{array}{c}\begin{array}{c}\text { Size } \\
(\mathrm{nm})\end{array} \\
230 \times 280\end{array}$} & \multicolumn{3}{|c|}{${ }^{17} \mathrm{O} /{ }^{16} \mathrm{O}\left(\times 10^{-4}\right)$} & \multicolumn{3}{|c|}{${ }^{18} \mathrm{O} /{ }^{16} \mathrm{O}\left(\times 10^{-3}\right)$} & \multirow{2}{*}{$\begin{array}{r}\text { Gr. } \\
1\end{array}$} & \multirow{2}{*}{$\begin{array}{l}\text { Min. } \\
\text { sil. }\end{array}$} \\
\hline & & & & 9.72 & \pm & 1.06 & 1.86 & \pm & 0.15 & & \\
\hline MET 00426 & ICM & MET_01B_11 & $280 \times 290$ & 8.46 & \pm & 0.98 & 1.73 & \pm & 0.14 & 1 & sil. \\
\hline MET 00426 & ICM & MET_01B_12 & $270 \times 280$ & 7.32 & \pm & 0.49 & 1.36 & \pm & 0.07 & 1 & sil. \\
\hline MET 00426 & ICM & MET_01B_15 & $165 \times 235$ & 5.96 & \pm & 0.48 & 1.56 & \pm & 0.08 & 1 & sil. \\
\hline MET 00426 & ICM & MET_01B_16 & $235 \times 260$ & 3.54 & \pm & 0.53 & 2.69 & \pm & 0.15 & 4 & sil. \\
\hline MET 00426 & ICM & MET_01B_20_1 & $290 \times 350$ & 7.72 & \pm & 0.76 & 1.74 & \pm & 0.11 & 1 & sil. \\
\hline MET 00426 & ICM & MET_01B_20_2 & $235 \times 280$ & 6.35 & \pm & 0.46 & 2.08 & \pm & 0.08 & 1 & sil. \\
\hline MET 00426 & ICM & MET_01B_28 & $235 \times 300$ & 3.50 & \pm & 0.58 & 2.93 & \pm & 0.16 & 4 & sil. \\
\hline MET 00426 & ICM & MET_01B_41 & $255 \times 315$ & 16.70 & \pm & 1.30 & 0.84 & \pm & 0.09 & 2 & sil. \\
\hline MET 00426 & ICM & MET_01B_50 & $200 \times 280$ & 5.59 & \pm & 0.59 & 1.38 & \pm & 0.09 & 1 & sil. \\
\hline MET 00426 & ICM & MET_01B_53_1 & $325 \times 445$ & 9.37 & \pm & 0.84 & 1.81 & \pm & 0.11 & 1 & sil. \\
\hline MET 00426 & ICM & MET_01B_53_2 & $260 \times 260$ & 5.82 & \pm & 0.45 & 2.09 & \pm & 0.08 & 1 & sil. \\
\hline MET 00426 & ICM & MET_01B_55 & $245 \times 280$ & 7.73 & \pm & 0.49 & 2.07 & \pm & 0.07 & 1 & sil. \\
\hline MET 00426 & ICM & MET_01B_61 & $350 \times 400$ & 21.90 & \pm & 0.33 & 2.31 & \pm & 0.30 & 1 & sil. \\
\hline MET 00426 & ICM & MET_01B_62 & $350 \times 420$ & 7.17 & \pm & 0.50 & 1.69 & \pm & 0.07 & 1 & sil. \\
\hline MET 00426 & ICM & MET_01B_65 & $300 \times 325$ & 3.74 & \pm & 0.43 & 2.85 & \pm & 0.12 & 4 & sil. \\
\hline MET 00426 & ICM & MET_01B_66 & $235 \times 280$ & 6.41 & \pm & 0.58 & 2.16 & \pm & 0.11 & 1 & sil. \\
\hline MET 00426 & ICM & MET_01B_67 & $235 \times 235$ & 7.02 & \pm & 0.62 & 2.01 & \pm & 0.10 & 1 & sil. \\
\hline MET 00426 & ICM & MET_01B_68 & $220 \times 220$ & 3.14 & \pm & 0.45 & 2.88 & \pm & 0.14 & 4 & sil. \\
\hline MET 00426 & FGR & MET_01A_8 & $210 \times 260$ & 5.19 & \pm & 0.30 & 1.81 & \pm & 0.06 & 1 & sil. \\
\hline MET 00426 & FGR & MET_01A_13 & $190 \times 190$ & 4.99 & \pm & 0.69 & 2.89 & \pm & 0.16 & 4 & sil. \\
\hline MET 00426 & FGR & MET_01A_22 & $260 \times 315$ & 5.12 & \pm & 0.26 & 1.97 & \pm & 0.05 & 1 & ox. \\
\hline MET 00426 & FGR & MET_01B_91 & $200 \times 220$ & 5.97 & \pm & 0.30 & 1.88 & \pm & 0.05 & 1 & sil. \\
\hline MET 00426 & FGR & MET_01D_1 & $210 \times 235$ & 5.51 & \pm & 0.72 & 1.22 & \pm & 0.11 & 1 & sil. \\
\hline MET 00426 & FGR & MET_01D_10 & $140 \times 235$ & 5.39 & \pm & 0.46 & 1.62 & \pm & 0.08 & 1 & sil. \\
\hline Acfer 094 & FGR & RC\#11_01_02 & $350 \times 210$ & 5.52 & \pm & 0.42 & 1.99 & \pm & 0.08 & 1 & sil. \\
\hline Acfer 094 & FGR & RC\#11_01_04 & $230 \times 165$ & 6.46 & \pm & 0.46 & 2.02 & \pm & 0.08 & 1 & sil. \\
\hline Acfer 094 & FGR & RC\#11_01_05 & $210 \times 165$ & 13.20 & \pm & 1.01 & 2.01 & \pm & 0.13 & 1 & sil. \\
\hline Acfer 094 & FGR & RC\#11_01_08 & $370 \times 260$ & 8.62 & \pm & 0.67 & 2.11 & \pm & 0.10 & 1 & sil. \\
\hline Acfer 094 & FGR & RC\#11_01_11 & $230 \times 210$ & 7.08 & \pm & 0.54 & 2.12 & \pm & 0.09 & 1 & sil. \\
\hline Acfer 094 & FGR & RC\#11_01_13 & $210 \times 260$ & 5.95 & \pm & 0.46 & 1.79 & \pm & 0.08 & 1 & sil. \\
\hline Acfer 094 & FGR & RC\#11_01_22 & $350 \times 400$ & 14.20 & \pm & 1.32 & 2.27 & \pm & 0.16 & 1 & sil. \\
\hline Acfer 094 & FGR & RC\#12_03 & $230 \times 280$ & 6.84 & \pm & 0.52 & 1.83 & \pm & 0.08 & 1 & sil. \\
\hline Acfer 094 & FGR & RC\#12_08 & $200 \times 235$ & 5.44 & \pm & 0.41 & 1.74 & \pm & 0.07 & 1 & sil. \\
\hline
\end{tabular}


684

685

686

687

688

689

690

\begin{tabular}{llllllllll}
\hline Grain & $\mathrm{O}$ & $\mathrm{Fe}$ & $\mathrm{Mg}$ & $\mathrm{Si}$ & $\mathrm{Al}$ & $\mathrm{S}$ & $\begin{array}{l}(\mathrm{Mg}+\mathrm{Fe}- \\
\mathrm{S}) / \mathrm{Si} *\end{array}$ & $\begin{array}{l}\mathrm{Mg} /(\mathrm{Mg}+\mathrm{Fe}- \\
\mathrm{S} *)\end{array}$ & type \\
\hline GR95_4_16 & 59.9 & 18.2 & 3.8 & 15.4 & 2.8 & $1.2 \pm 0.2$ & 0.20 & px-like \\
GR95_8_7 & 56.1 & 8.2 & 4.5 & 11.1 & 14.1 & 3.0 & $0.9 \pm 0.1$ & 0.46 & px-like \\
GR95_12_5 & 56.8 & 16.7 & 6.2 & 16.2 & & 4.0 & $1.2 \pm 0.2$ & 0.33 & px-like \\
GR95_12_6 & 58.9 & 5.8 & 18.8 & 16.4 & & & $1.5 \pm 0.2$ & 0.76 & intermed. \\
GR95_13_22 & 61.7 & 6.0 & 19.4 & 13.0 & & & $2.0 \pm 0.3$ & 0.76 & ol-like \\
GR95_13_24 & 55.5 & 19.7 & 7.3 & 13.4 & 4.1 & $1.7 \pm 0.3$ & 0.32 & ol-like \\
GR95_13_27 & 51.7 & 9.7 & 19.6 & 15.5 & 3.4 & $1.7 \pm 0.1$ & 0.76 & compound \\
GR95_13_29 & 58.1 & 11.5 & 9.3 & 19.6 & 1.6 & $1.0 \pm 0.1$ & 0.45 & compound \\
GR95_14_13 & 52.4 & 7.5 & 10.0 & 27.2 & 2.9 & $0.5 \pm 0.1$ & 0.68 & Si-rich \\
EET_02A_B_2 & 55.0 & 7.5 & 27.1 & 10.5 & & $3.3 \pm 0.4$ & 0.78 & Si-poor \\
EET_02A_B_3 & 61.0 & 8.7 & 14.5 & 15.9 & & $1.5 \pm 0.2$ & 0.62 & intermed. \\
EET_02A_B_10 & 57.0 & 14.8 & 14.0 & 14.3 & & $2.0 \pm 0.3$ & 0.49 & ol-like \\
EET_02A_B_31 & 58.8 & 12.2 & 12.2 & 14.8 & 2.0 & $1.5 \pm 0.2$ & 0.50 & intermed. \\
EET_02B_2 & 58.7 & 18.7 & 4.9 & 17.7 & & $1.3 \pm 0.2$ & 0.21 & intermed. \\
EET_02B_9 & 61.5 & 21.1 & 5.2 & 12.2 & & $2.2 \pm 0.3$ & 0.20 & ol-like \\
EET_5B_5 & 55.4 & 16.3 & 13.9 & 11.8 & 2.7 & $2.3 \pm 0.2$ & 0.50 & Si-poor \\
EET_6_6 & 54.4 & 19.2 & 7.9 & 16.2 & 2.4 & $1.5 \pm 0.2$ & 0.32 & intermed. \\
EET_6_13 & 54.4 & 17.4 & 8.0 & 16.0 & 4.2 & $1.3 \pm 0.2$ & 0.38 & intermed. \\
EET_6_15 & 56.6 & 16.4 & 14.9 & 12.2 & & $2.6 \pm 0.3$ & 0.48 & Si-poor \\
\hline *Both ratios have been corrected for the presence of S in the grains. Assuming that sulfur is present as FeS, the S abundances \\
have been subtracted directly from the measured Fe abundances. & & & &
\end{tabular}

Table 4. Elemental compositions of presolar silicate grains from GRA 95229 and EET 92161

determined by Auger electron spectroscopy (AES). Relative abundance errors are $3.6 \%(\mathrm{O})$, 11.2\% (Fe), 9.4\% (Mg), 11\% (Si), 24.9\% (Al), and 10\% (S). "px-like" and "ol-like" stand for pyroxene- and olivine-like grain stoichiometries $((\mathrm{Mg}+\mathrm{Fe}-\mathrm{S} / \mathrm{Si}) \sim 1$ and $\sim 2)$, respectively. "intermed." denotes grains with intermediate ratios of $\sim 1.3$ to 1.7 , while "Si-rich" and "Sipoor" characterize grains with respective ratios $>2$ and $<1$. "Compound" denotes grains that

consist of sub-grains with significantly different $(\mathrm{Mg}+\mathrm{Fe}-\mathrm{S}) / \mathrm{Si}$ and/or $\mathrm{Mg} /(\mathrm{Mg}+\mathrm{Fe}-\mathrm{S})$ ratios. 


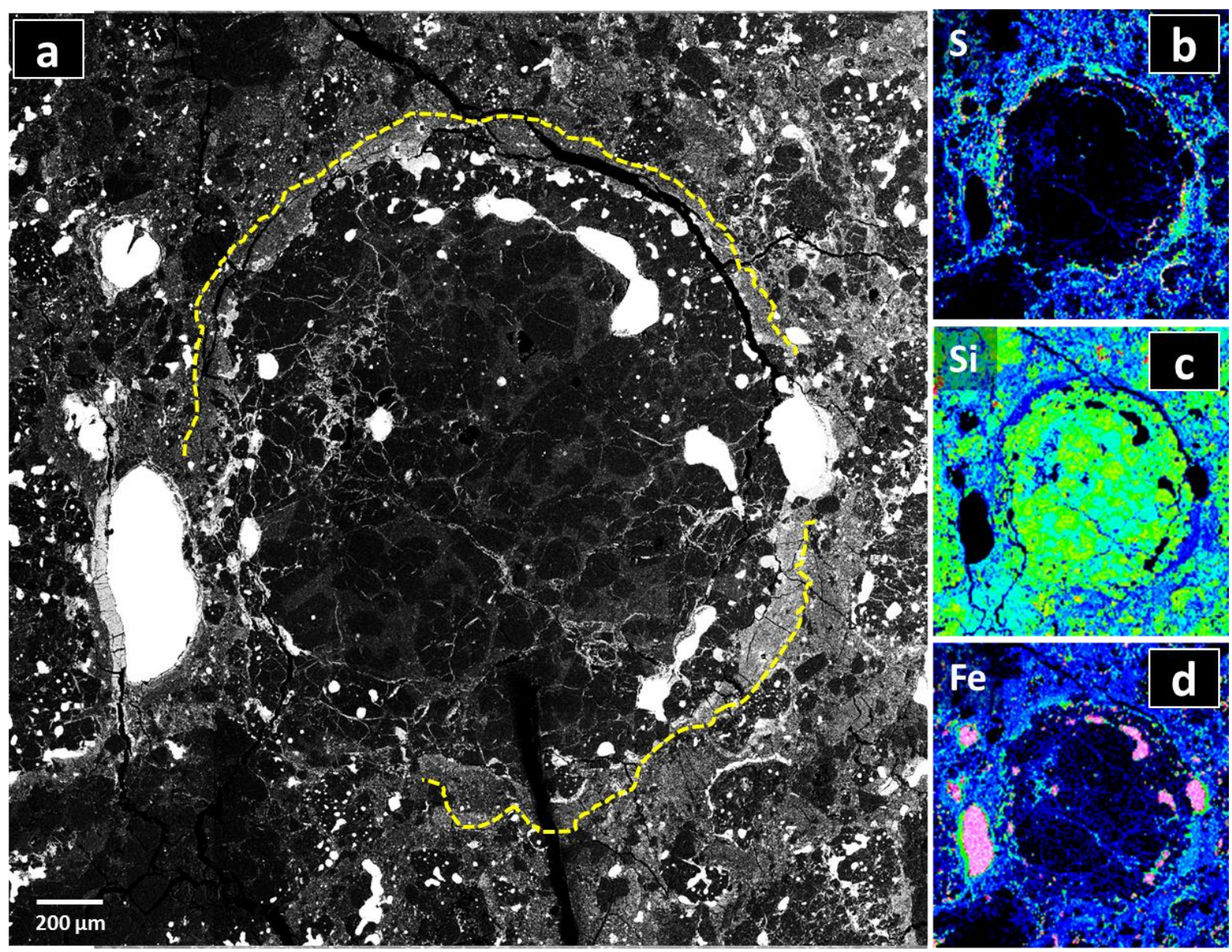

695 Figure 1: (a) BSE- and X-ray elemental maps of (b) S, (c) Si and (d) Fe of a rimmed

696 chondrule in GRA 95229. The FGR contains the presolar grains GR9513_6, GR95_13_22,

697 GR95_13_24, GR95_13_27, GR95_13_29, and GR95_14_13 (Table 2). The dashed yellow

698 line in panel (a) marks the outline of the FGR, which can also be identified from the elemental

699 maps in $(b-d)$. 


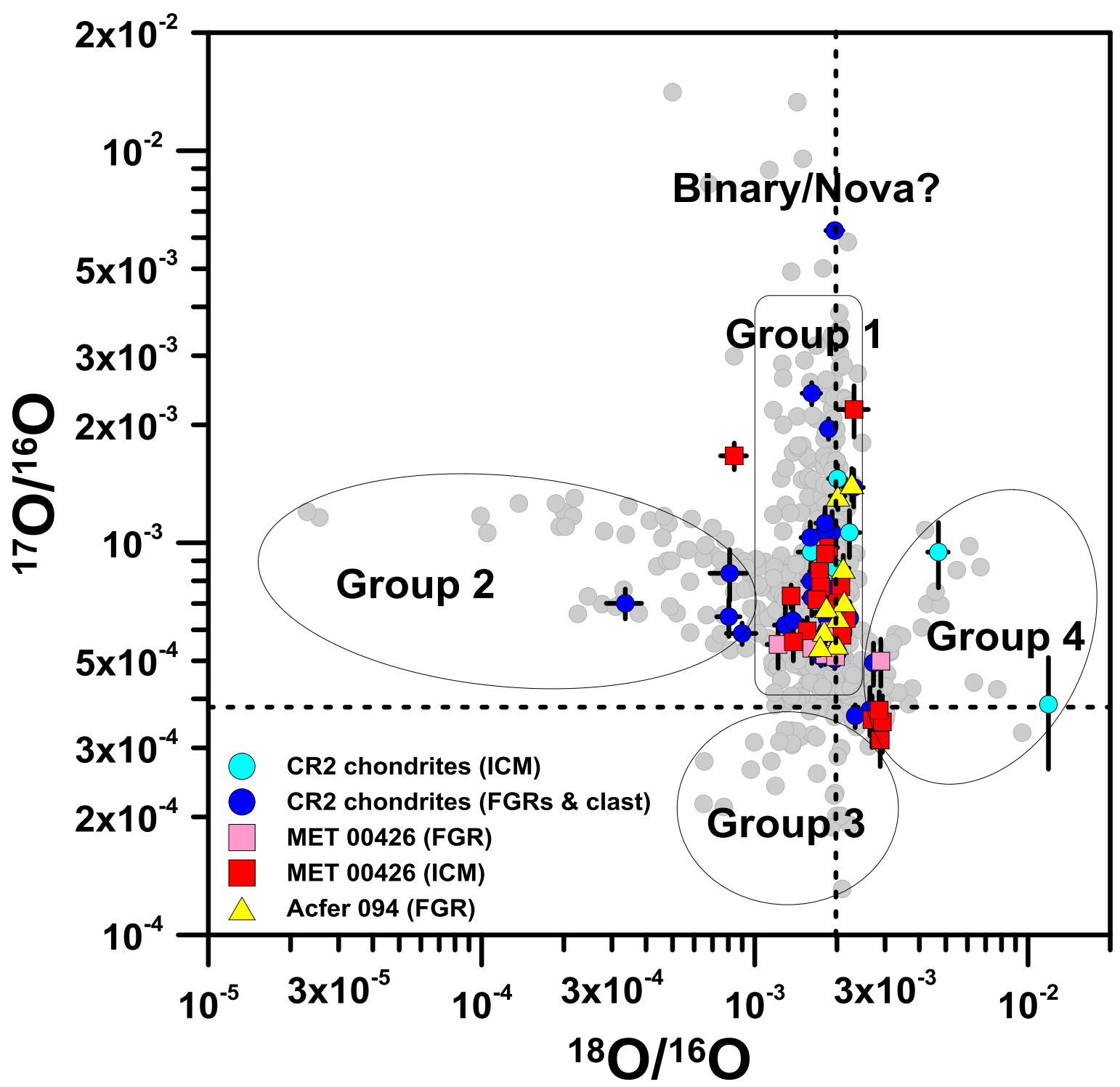

701

702

703

Figure 2. Oxygen-isotopic ratios of presolar O-anomalous grains (silicates and oxides) identified in FGRs and one matrix-like clast (blue symbols) as well as in the ICM of the five CR chondrites investigated in this study. Grain groups (NITTLER et al. 1997, 2008) are symbolized by ellipses. For comparison, data for presolar silicates and oxides (gray symbols) from BLAND et al. 2007; Bose et al. 2010, 2012; CHOI et al. 1998; Floss \& STADERMANN 2009, 2012; LeITNER et al. 2012; MosteFAOUI \& HoPPE 2004; NAGASHIMA et al. 2004; NGUYEN et al. 2007, 2010; NITTLER et al. 1997, 2008; VOLLMER et al. 2009a, 2009b; ZHAO et

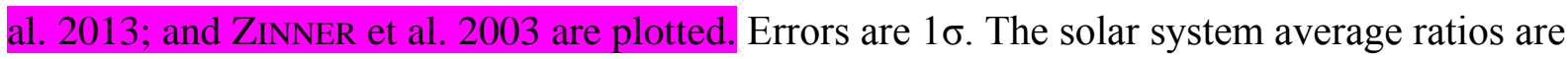
indicated by the dashed lines. 


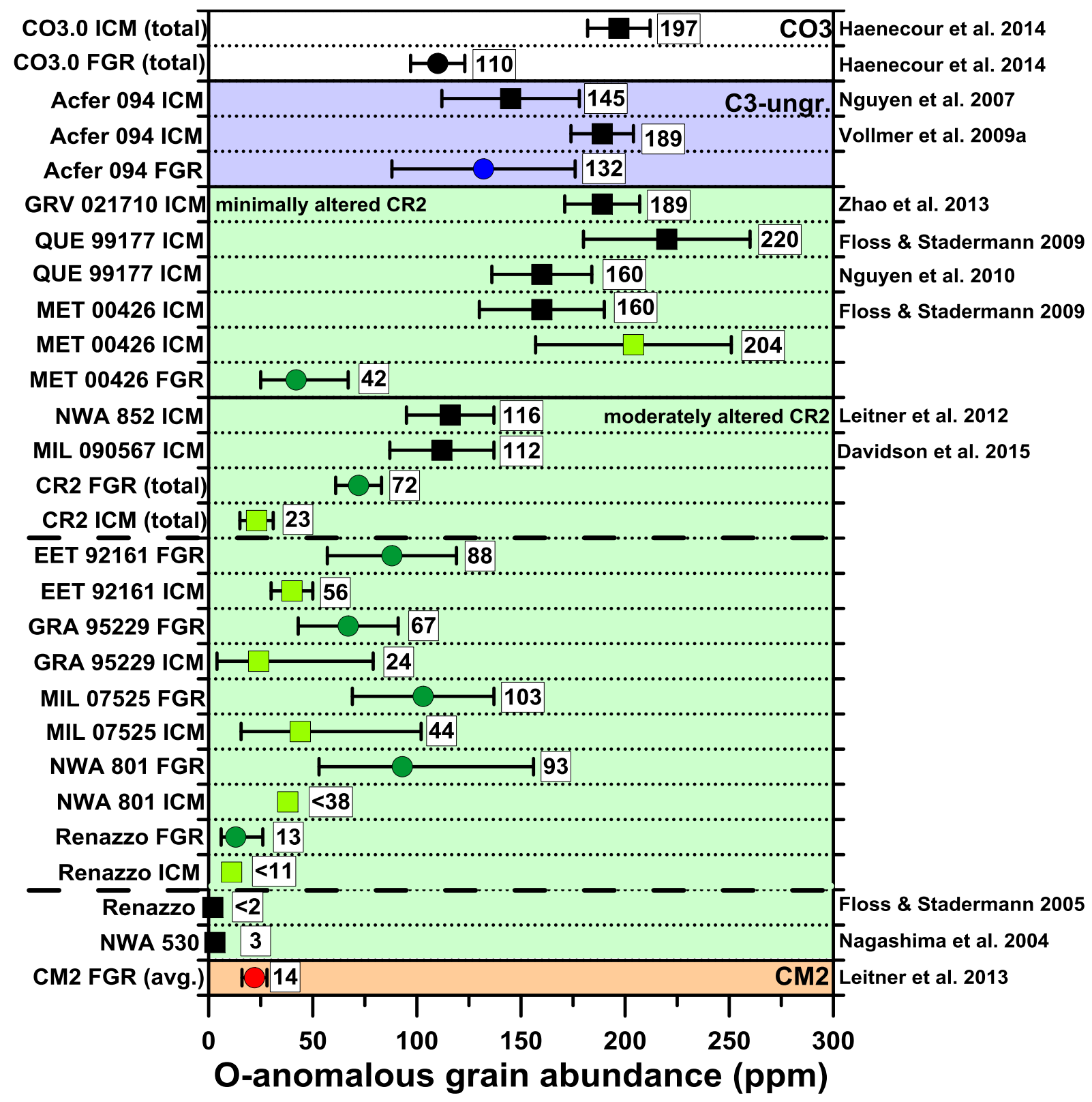

Figure 3. Abundances (in ppm) of presolar O-rich dust grains for the CR chondrites and Acfer 094 investigated in this study (green and blue symbols) in comparison with literature data for $\mathrm{CM}, \mathrm{CR}$ and $\mathrm{CO}$ chondrites, as well as the ungrouped carbonaceous chondrite Acfer 094. All errors are $1 \sigma$. 


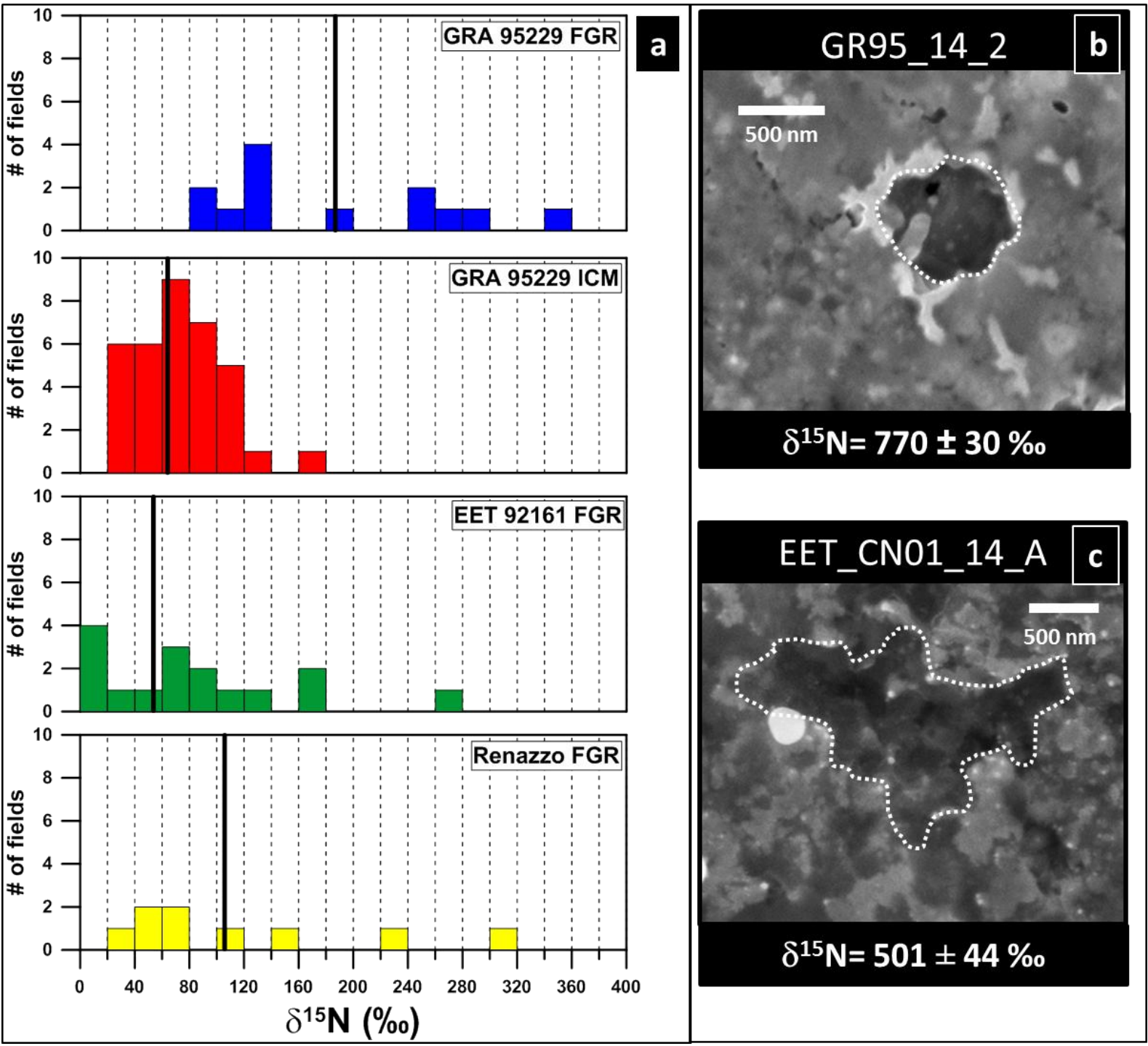

Figure 4. (a) Nitrogen isotopic compositions of individual measurement fields $\left(10 \times 10 \mu \mathrm{m}^{2}\right)$ in FGRs from GRA 95229, EET 92161, Renazzo, and in the ICM of GRA 95229. The Nisotopic compositions are displayed as $\delta$-values, i.e., per mil deviations from the terrestrial value $\left({ }^{15} \mathrm{~N} /{ }^{14} \mathrm{~N}=3.676 \times 10^{-3}\right)$. The bold lines in each plot mark the respective weighted average $\delta^{15} \mathrm{~N}$. (b) SEM micrograph showing localized spots of organic material with anomalous $\mathrm{N}$-isotopic composition (marked by the white dotted lines; data not shown in (a)) in a FGR from GRA 95229. (c) SEM micrograph showing localized spots of organic material with anomalous $\mathrm{N}$-isotopic composition (marked by the white dotted lines; data not shown in (a)) in a FGR from EET 92161 are displayed. All errors are $1 \sigma$. 


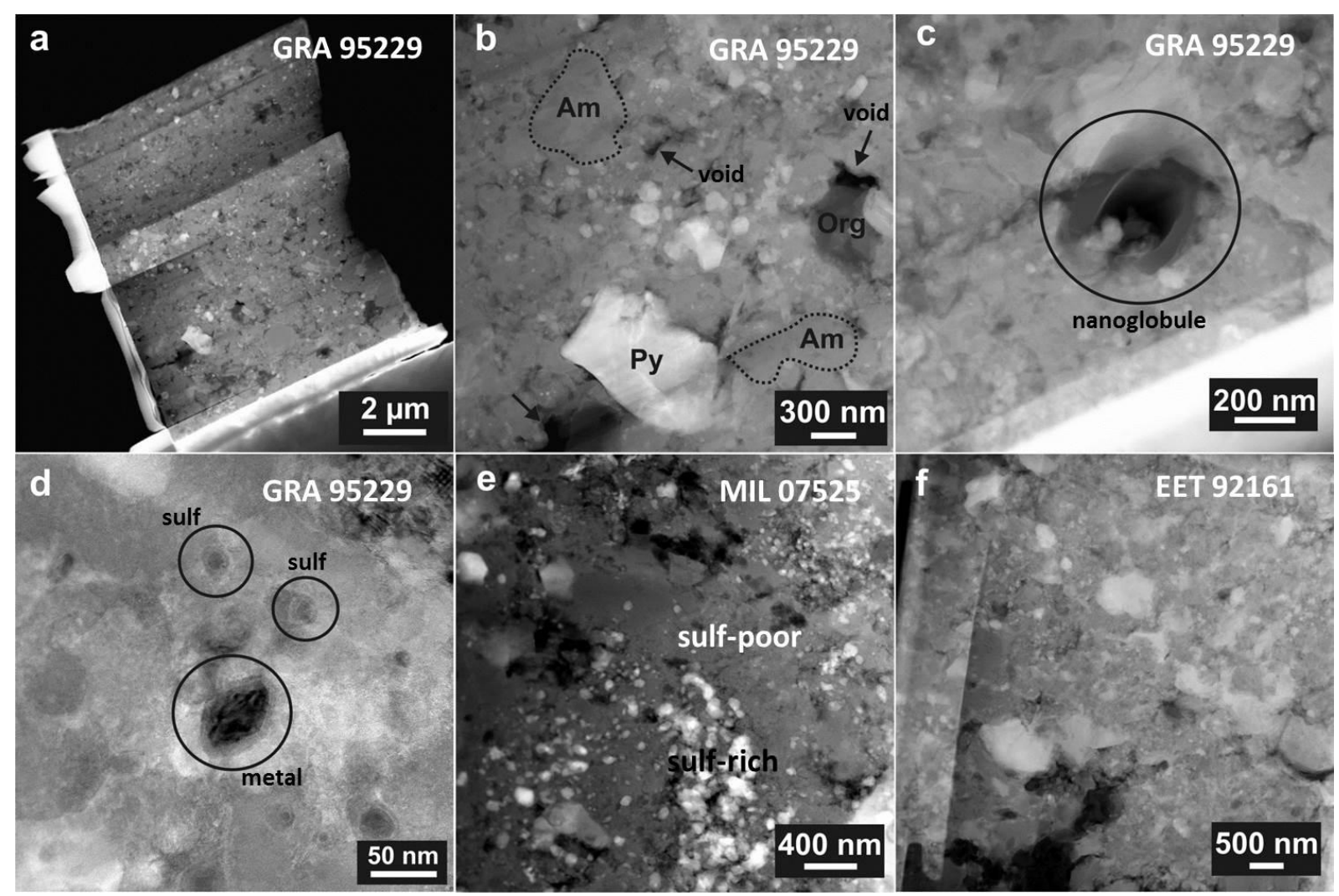

731 Figure 5. TEM images of FIB lamellae from FGRs in GRA 95229, EET 92161, and MIL

732 07525. (a) HAADF (high-angle annular dark-field) image from GRA. (b) Close-up HAADF

733 image from GRA with pyrrhotite (Py), organic matter (Org), amorphous silicates (Am) and

734 voids (arrow-marked). (c) Close-up HAADF image of a hollow organic nanoglobule from

735 GRA. (d) Close-up bright-field image with sub-micrometer-sized sulfide and metal grains

736 exhibiting reaction rims (encircled) from GRA. (e) HAADF image of the MIL lamella with

737 distinct sulfide-rich (bright) and -poor (dark) areas. (f) HAADF image from EET showing 738 major signs of recrystallization (upper half) as low-porosity Fe-enriched (bright) areas. 

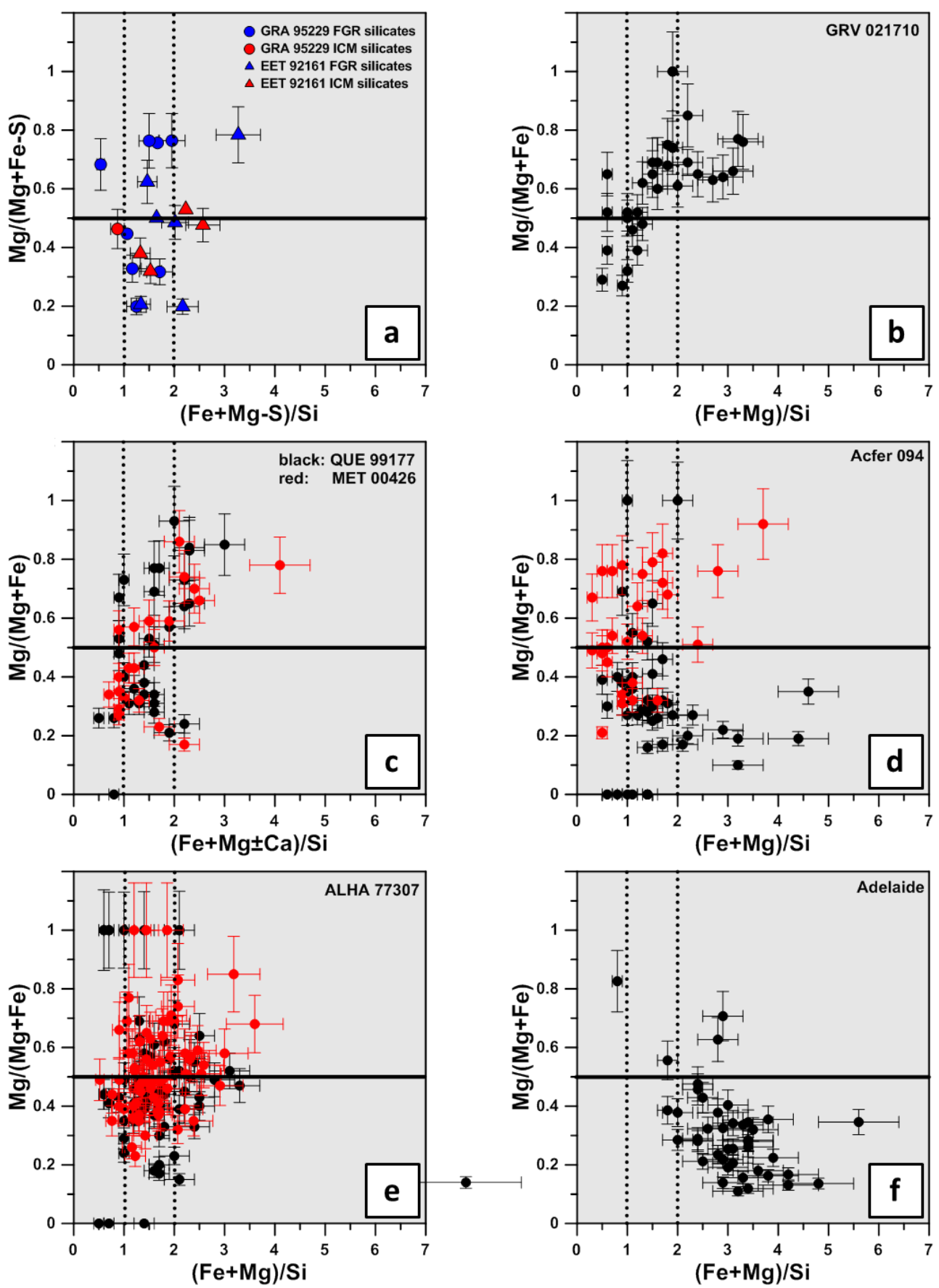

Figure 6. (a) Mg-Fe-Si element abundances for presolar silicates from FGRs and ICM in GRA 95229 and EET 92161, (b) presolar silicate data from GRV 021710 (CR) (ZHAO et al. 2013), (c) QUE 99177 and MET 00426 (both CR) (Floss \& STADERMAnN 2009), (d) Acfer 094 (C-ungrouped) (Vollmer et al. 2009a, BosE et al. 2010), (e) ALHA 77307 (CO3.0)

745 (NGuYen et al. 2010, Bose et al. 2012), (f) and Adelaide (C-ungrouped) (Floss \& 746 StAdermann 2012) (f). All errors are $1 \sigma$. "Fe+Mg $-\mathrm{S}$ " means that the ratios have been corrected for the presence of $\mathrm{S}$. Dotted vertical lines denote the compositions of 
748 stoichiometric pyroxene (1) and olivine (2). The horizontal lines mark the border between 749 Mg-rich and Fe-rich compositions. 